\title{
Optimal design under the one-dimensional wave equation
}

\author{
FAUSTINO MAESTRE ${ }^{\dagger}$ \\ Departamento de Matemáticas, ETSI Industriales, Universidad de Castilla - La Mancha, \\ 13071 Ciudad Real, Spain \\ ARNAUD MÜNCH ${ }^{\ddagger}$ \\ Laboratoire de Mathématiques de Besançon, Université de Franche-Comté, \\ 16, route de Gray, 25030 Besançon, France \\ PABLO PEDREGAL ${ }^{\S}$ \\ Departamento de Matemáticas, ETSI Industriales, Universidad de Castilla - La Mancha, \\ 13071 Ciudad Real, Spain
}

[Received 5 July 2006 and in revised form 26 September 2007]

\begin{abstract}
An optimal design problem governed by the wave equation is examined in detail. Specifically, we seek the time-dependent optimal layout of two isotropic materials on a 1-d domain by minimizing a functional depending quadratically on the gradient of the state with coefficients that may depend on space, time and design. Typically, such problems are ill-posed in the sense that there is no optimal design. We therefore examine relaxation by using the representation of two-dimensional $\left((x, t) \in \mathbb{R}^{2}\right)$ divergence free vector fields as rotated gradients. By means of gradient Young measures, we transform the original optimal design problem into a non-convex vector variational problem, for which we can compute an explicit form of the "constrained quasiconvexification" of the cost density. Moreover, this quasiconvexification is recovered by first or second order laminates which give us the optimal microstructure at every point. Finally, we analyze the relaxed problem, and some numerical experiments are performed. The perspective is similar to the one developed in previous papers for linear elliptic state equations. The novelty here lies in the state equation (the wave equation), and our contribution consists in understanding the differences with respect to elliptic cases.
\end{abstract}

\section{Introduction}

Optimal design problems in conductivity and elasticity have been extensively studied from various perspectives. For the homogenization viewpoint, see [1]. For more simulation-oriented approaches, see [4, 9]. For treatments based on variational reformulations, see [23]. In many of these examples, the state equation is assumed to be isotropic. There has also been attempts to understand nonisotropic situations ([20] and references therein).

Suppose we choose two diagonal, non-isotropic, $2 \times 2$ matrices of the form

$$
A_{\alpha}=\left(\begin{array}{cc}
1 & 0 \\
0 & \alpha
\end{array}\right), \quad A_{\beta}=\left(\begin{array}{cc}
1 & 0 \\
0 & \beta
\end{array}\right)
$$

\footnotetext{
E-mail: Faustino.Maestre@uclm.es

E-mail: arnaud.munch@univ-fcomte.fr

§E-mail: pablo.pedregal@uclm.es
} 
and consider the state equation

$$
\operatorname{div}\left(\left[\chi(x) A_{\alpha}+(1-\chi(x)) A_{\beta}\right] \nabla u\right)=0 \quad \text { in } \Omega,
$$

where $\Omega \subset \mathbb{R}^{2}$ is a bounded, regular, simply connected domain. It is easy to see that we can also write

$$
\operatorname{div}\left(\left(\begin{array}{lc}
1 & 0 \\
0 & \chi(x) \alpha+(1-\chi(x)) \beta
\end{array}\right) \nabla u\right)=0 \quad \text { in } \Omega
$$

and even more so

$$
\operatorname{div}\left(u_{x_{1}},[\chi(x) \alpha+(1-\chi(x)) \beta] u_{x_{2}}\right)=0 .
$$

In the case where $\Omega=(0, T) \times(0,1)$, and we take both $\alpha$ and $\beta$ negative, we see that we have a $1-\mathrm{d}$ wave equation as state equation, and 2-d optimal designs can be interpreted as 1-d time-dependent optimal designs. For this reason, we change the notation and write $x\left(=x_{2}\right)$ for the spatial variable, $t\left(=x_{1}\right)$ for the time variable, and replace $\alpha, \beta$ by $-\alpha,-\beta$, respectively, so that we focus on such a wave equation. We also change accordingly the domain, and consider initial and boundary conditions as is usual in hyperbolic problems. Yet notice that the non-isotropic elliptic example is also contained in our analysis.

Problems with optimal control in the coefficients are rather well-known in the elliptic case. Homogenization has been the main tool to deal with these when cost functionals do not depend on derivatives of the state. As indicated earlier, [1] is still, as far as we can tell, an up-to-date reference for the use of homogenization in optimal design problems. Our interest in understanding optimal design problems for cost functionals depending on gradients of the state led us to explore the use of gradient Young measures in this kind of problems ([23], [24]). See [27] for a pioneering situation that is quite instructive to better understand how different optimal design problems with quadratic cost functionals in the gradient can be. Other references that have treated similar situations from the perspective of homogenization are [11], [14]. Our perspective does not require a full understanding of the $G$-closure problem (as in homogenization) as the emphasis is not placed on the tensors that can be obtained by mixtures (the $G$-closure), but rather on the set of pairs of vectors that can be related through some tensor of the $G$-closure. Hence, although intimately connected to homogenization, our approach focuses directly on pairs of fields that can occur in relaxed state equations. In this way, we can treat cost functionals depending on the gradient of the state directly without further ingredients.

Since, to some degree, the elliptic situation in the conductivity setting is understood (even numerical simulations of optimal microstructures have been produced as in [9]), a next natural step is to examine the same optimal design problems with quadratic cost functionals on the gradient of the state under a hyperbolic state equation, so as to better understand the differences introduced in the analysis and on the numerics because of this hyperbolic nature. One issue here is the phenomenon of concentration of cost (energy). It is well-understood that Young measures cannot capture concentration effects. We easily resolve this issue by demanding some extra regularity on initial data. Notice that if designs were not allowed to vary with time, the situation would be much simpler as it would not require "homogenization" or the formation of microstructure on this time variable. Many of the standard homogenization facts could be used and exploited. Indeed, one of our main goals is to grasp this dependence of designs on time.

Except for the works of Lurie ([15], [16], [17]), this sort of problems have not been addressed in the literature. In particular, he has been investigating over the years the analogue of the $G$-closure 
for dynamic designs. Being motivated by realistic applications, a main concern in his work is to understand the relationship and interaction between the dynamic nature of the problem and the "dynamics" of microstructure. In a sense, even in a static situation, microstructure (laminates) is something dynamic as it is a never-ending refinement process. When this process interacts with real time, some funny situations may occur (including the formation of shocks). This is well-documented for instance in [17], where restrictions on the velocity of formation of microstructure and the dynamics of the state equation are explicitly given so that undesirable behavior is ruled out. We have avoided this issue altogether as we model dynamic microstructure through families of probability measures (Young measures) depending both on space and time so that there is only one dynamic process associated with time. Even so, it is interesting to stress that the laminates we get in our numerical simulations (Section 7) satisfy these requirements in the best way possible as they are oriented parallel to the time axis. This is the most favorable situation in Lurie's work. See also the comments after Conjecture 1 Another main difference of our work and that of Lurie is that we are interested from the beginning in a cost functional which is quadratic in the gradient of the state. Our methods allow us to treat directly this sort of problem without understanding first the $G$-closure set. A main concern in the work of Lurie is to better understand the $G$-closure set corresponding to a dynamical situation, and, in particular, to discover the differences with respect to its elliptic counterpart.

Other works dealing with optimal control problems under the wave equation in greater dimensions can be found in [6], where the control is a time-dependent coefficient, and under other constraints on modes where there is vibration. In this sense another work in which the authors examine time-harmonic solutions of the wave equation is [3], where they prove a relaxation result for this problem and very interesting results on existence of classical solutions for some particular cases. In the wave equation literature, we can find a huge family of optimal control problems where the design variable is not in the highest derivative term. When the control term acts on the first order derivative in time, the term is known as a "damping" term. These problems are of a different nature physically as well as mathematically. Some relevant references on this topic are [5, 10, 12].

\subsection{Problem statement}

We will thus study the following optimal design problem. We consider a design domain $\Omega=$ $(0,1) \subset \mathbb{R}$, a positive time $T>0$, and a maximum amount $V_{\alpha} \in(0,1)$ of one material at our disposal. The optimal design problem consists in deciding, for each time $0<t<T$, the best distribution in $\Omega$ of the two materials in order to minimize the time-dependent cost functional depending on the square of the gradient (with respect to both variables $(t, x)$ ) of the underlying state. More precisely, let us denote by $(P)$ the problem that consists in minimizing

$$
\text { (P) } \quad I(\chi)=\int_{0}^{T} \int_{\Omega}\left[u_{t}^{2}(t, x)+a(t, x, \chi) u_{x}^{2}(t, x)\right] \mathrm{d} x \mathrm{~d} t
$$

where $u$ is the unique solution of

$$
\begin{array}{ll}
u_{t t}-\operatorname{div}\left([\alpha \chi+\beta(1-\chi)] u_{x}\right)=0 & \text { in }(0, T) \times(0,1), \\
u(0, x)=u_{0}(x), \quad u_{t}(0, x)=u_{1}(x) & \text { in } \Omega, \\
u(t, 0)=f(t), \quad u(t, 1)=g(t) & \text { in }[0, T],
\end{array}
$$

and the functions $a, u_{0}$ and $u_{1}$ are known. We have put a vanishing source term for simplicity. The function $\chi \in L^{\infty}([0, T] \times \Omega ;\{0,1\})$ is the design variable, and it indicates where we place the 
$\alpha$-material for each time $t$. Since $\chi$ is a binary variable, $a(t, x, \chi) \in\{a(t, x, 0), a(t, x, 1)\}$, we can write

where

$$
a(t, x, \chi)=\chi(t, x) a_{\alpha}(t, x)+(1-\chi(t, x)) a_{\beta}(t, x),
$$

$$
a_{\alpha}(t, x)=a(t, x, 1), \quad a_{\beta}(t, x)=a(t, x, 0) .
$$

In addition, we make the assumption $0<\alpha<\beta$, and

$$
a_{\alpha}(t, x)+\alpha \geqslant 0, \quad a_{\beta}(t, x)+\beta \geqslant 0 .
$$

The amount of the $\alpha$-material is given, and therefore we have to enforce the volume constraint

$$
\int_{\Omega} \chi(t, x) \mathrm{d} x \leqslant V_{\alpha}|\Omega|, \quad \forall t \in[0, T] .
$$

The lack of classical solutions for such problems is well understood (see [22, Theorem 11]). In this sense we propose and analyze a relaxation of the problem.

Our approach is based on an equivalent variational reformulation of the original optimal design problem as a non-convex vector variational problem. As in other situations examined from this perspective [2, 23], we replace a scalar problem with differential constraints by a vector variational problem with integral constraints (where the state equation is implicit in the new cost function). It is well-known that the non-existence of optimal solution for a vector variational problem is intimately associated with the lack of quasiconvexity of the cost functional, and in this sense we propose to analyze the "constrained quasiconvexification" for this last problem by using gradient Young measures as generalized solutions of variational problems. We compute an explicit relaxation of the original optimal design problem in the form of a relaxed (quasiconvexified) variational problem.

It is elementary to check (this is done with some detail in Section 2) the equivalence of our dynamic optimal design problem with the following non-convex, vector variational problem:

$$
\min _{U} \hat{I}(U)=\int_{0}^{T} \int_{\Omega} W(t, x, \nabla U(t, x)) \mathrm{d} x \mathrm{~d} t
$$

subject to

$$
\begin{aligned}
& U=\left(U^{(1)}, U^{(2)}\right) \in H^{1}([0, T] \times \Omega)^{2}, \\
& U^{(1)}(0, x)=u_{0}(x), \quad U_{t}^{(1)}(0, x)=u_{1}(x) \quad \text { in } \Omega, \\
& U^{(1)}(t, 0)=f(t), \quad U^{(1)}(t, 1)=g(t) \quad \text { in }[0, T], \\
& \int_{\Omega} V(t, x, \nabla U(t, x)) \mathrm{d} x \leqslant V_{\alpha}|\Omega|, \quad \forall t \in[0, T] .
\end{aligned}
$$

The two integrands involved are

$$
\begin{aligned}
& W(t, x, A)= \begin{cases}a_{11}^{2}+a_{\alpha}(t, x) a_{12}^{2} & \text { if } A \in \Lambda_{\alpha}, \\
a_{11}^{2}+a_{\beta}(t, x) a_{12}^{2} & \text { if } A \in \Lambda_{\beta} \backslash \Lambda_{\alpha}, \\
+\infty & \text { else, }\end{cases} \\
& V(t, x, A)= \begin{cases}1 & \text { if } A \in \Lambda_{\alpha}, \\
0 & \text { if } A \in \Lambda_{\beta} \backslash \Lambda_{\alpha}, \\
+\infty & \text { else. }\end{cases}
\end{aligned}
$$

Here

$$
\Lambda_{\gamma}=\left\{A \in M^{2 \times 2}: M_{-\gamma} A^{(1)}-R A^{(2)}=0\right\}, \quad \gamma=\alpha, \beta,
$$


where $A^{(i)}$ is the $i$-th row of the matrix $A=\left(\begin{array}{ll}a_{11} & a_{12} \\ a_{21} & a_{22}\end{array}\right)$, and

$$
M_{-\gamma}=\left(\begin{array}{cc}
1 & 0 \\
0 & -\gamma
\end{array}\right), \quad R=\left(\begin{array}{cc}
0 & -1 \\
1 & 0
\end{array}\right) \text {. }
$$

\subsection{Results}

To write down an explicit relaxation, put

$$
h(t, x)=\beta a_{\alpha}(t, x)-\alpha a_{\beta}(t, x)
$$

and for $F=\left(\begin{array}{ll}F_{11} & F_{12} \\ F_{21} & F_{22}\end{array}\right), s \in \mathbb{R}$, set

$$
\psi(F, s)=F_{12} F_{21}+\frac{\alpha}{s(\beta-\alpha)^{2}}\left(\beta F_{12}+F_{21}\right)^{2}+\frac{\beta}{(1-s)(\beta-\alpha)^{2}}\left(\alpha F_{12}+F_{21}\right)^{2} .
$$

Consider the variational problem

$$
(R P) \quad \min _{U, s} \int_{0}^{T} \int_{\Omega} \varphi(t, x, \nabla U(t, x), s(t, x)) \mathrm{d} x \mathrm{~d} t
$$

subject to

$$
\begin{aligned}
& U \in H^{1}([0, T] \times \Omega)^{2}, \quad \operatorname{tr}(\nabla U(t, x))=0, \\
& U^{(1)}(0, x)=u_{0}(x), \quad U_{t}^{(1)}(0, x)=u_{1}(x) \quad \text { in } \Omega, \\
& U^{(1)}(t, 1)=f(t), \quad U^{(1)}(t, 0)=g(t) \quad \text { in }[0, T], \\
& 0 \leqslant s(t, x) \leqslant 1, \quad \int_{\Omega} s(t, x) \mathrm{d} x \leqslant V_{\alpha}|\Omega| \quad \forall t \in[0, T],
\end{aligned}
$$

where $\varphi(t, x, F, s)$ is explicitly given by the surprising expression

$$
\left\{\begin{array}{cc}
\frac{h}{s \beta(\beta-\alpha)^{2}}\left(\beta^{2}\left|F_{12}\right|^{2}+\left|F_{21}\right|^{2}+2 \beta F_{12} F_{21}\right)+\left|F_{11}\right|^{2}-\frac{a_{\beta}}{\beta} F_{12} F_{21} \\
& \text { if } h(x, t) \geqslant 0, \psi(F, s) \leqslant 0, \\
\frac{-h}{(1-s) \alpha(\beta-\alpha)^{2}}\left(\alpha^{2}\left|F_{12}\right|^{2}+\left|F_{21}\right|^{2}+2 \alpha F_{12} F_{21}\right)+\left|F_{11}\right|^{2}-\frac{a_{\alpha}}{\alpha} F_{12} F_{21}, & \text { if } h(x, t) \leqslant 0, \psi(F, s) \leqslant 0, \\
-\operatorname{det} F+\frac{1}{s(1-s)(\beta-\alpha)^{2}}\left(\left((1-s) \beta^{2}\left(\alpha+a_{\alpha}\right)+s \alpha^{2}\left(\beta+a_{\beta}\right)\right)\left|F_{12}\right|^{2}\right. \\
\left.+\left((1-s)\left(\alpha+a_{\alpha}\right)+s\left(\beta+a_{\beta}\right)\right)\left|F_{21}\right|^{2}+2\left(\left(\alpha+a_{\alpha}\right) \beta-s h\right) F_{12} F_{21}\right) & \text { if } \psi(F, s) \geqslant 0 .
\end{array}\right.
$$

tr stands for the trace of a matrix. All that matters is that this integrand $\varphi$ is known in closed form.

THEOREM 1 Suppose that the initial data $u_{0}$ and $u_{1}$ have the regularity

$$
u_{0} \in H^{2}(0,1) \cap H_{0}^{1}(0,1), \quad u_{1} \in H_{0}^{1}(0,1) .
$$

Then the variational problem $(R P)$ is a relaxation of the initial optimization problem $(P)$ in the sense that 
(a) the infima of both problems coincide;

(b) there are optimal solutions for the relaxed problem $(R P)$;

(c) these solutions codify (in the sense of Young measures) the optimal microstructures of the original optimal design problem.

For the interpretation of Young measure solutions in this statement, we refer the reader to the already mentioned contributions in the elliptic case. It is closely related to relaxation in vector, non-convex variational problems $([\overline{8}])$. These optimal Young measures carry the information on optimal microstructures, both on the local distribution of materials, and on the geometry of optimal microarrangements. See more on this interpretation in Section 7.

In addition, we can provide optimal microstructures explicitly.

THEOREM 2 Optimal, dynamic microarrangements of the two materials leading to the relaxed formulation are always laminates which can be given in a completely explicit form.

The formulae for all these laminates are given at the end of Section 4, where we compute these optimal microstructures corresponding to first and second order laminates.

The main new contribution here is therefore to understand the character of the hyperbolic state law, and the differences it introduces with respect to the better known elliptic case. Some of these differences are related to the fact that the manifolds $\Lambda_{\gamma}$ are two 2-dimensional subspaces whose intersection is another 1-dimensional manifold. Moreover, there are rank-one connections within those manifolds. An interesting consequence is that the relaxed integrand is finite everywhere (except for the condition involving the trace) in contrast with the elliptic case where the relaxed integrand is finite only in a certain (quasi)convex subset. An important issue is that optimal Young measures gives us the necessary information about the behavior of minimizing sequences of the original optimal design problem.

A subsequent important step is to explore the relaxed problem $(R P)$ in some particular cases, like the ones described in Section 5, with the objective of producing numerical simulations of optimal time-dependent structures [18]. For some particular situations in the (static) elliptic case, it has been shown that a simple relaxation consists in replacing the original discrete design variable $\chi \in L^{\infty}(\Omega ;\{0,1\})$ by its convex envelope $s \in L^{\infty}(\Omega ;[0,1])$. For the (dynamic) hyperbolic case with $a_{\alpha}=a_{\beta}=1$, some numerical experiments (see Section 7) suggest that the above assertion is true. In this regard, we make the following conjecture (examined briefly in Section 6).

Conjecture 1 Suppose $a_{\alpha}=1, a_{\beta}=1$. The optimization problem

$$
(\widetilde{R P}) \quad \min _{s} \tilde{I}(s)=\int_{0}^{T} \int_{\Omega}\left[u_{t}^{2}(t, x)+u_{x}^{2}(t, x)\right] \mathrm{d} x \mathrm{~d} t,
$$

where $u$ is the unique solution of

$$
\begin{aligned}
& u_{t t}-\operatorname{div}\left([\alpha s+\beta(1-s)] u_{x}\right)=0 \quad \text { in }(0, T) \times(0,1), \\
& u(0, x)=u_{0}(x), \quad u_{t}(0, x)=u_{1}(x) \quad \text { in } \Omega, \\
& u(t, 0)=f(t), \quad u(t, 1)=g(t) \quad \text { in }[0, T], \\
& \int_{\Omega} s(t, x) \mathrm{d} x \leqslant V_{\alpha}|\Omega|, \quad \forall t \in[0, T], \\
& 0 \leqslant s(t, x) \leqslant 1,
\end{aligned}
$$

is equivalent to the original optimal design problem $(P)$ in the sense that 
(a) the infima of both problems coincide, i.e., $\inf (\widetilde{R P})=\inf (P)$;

(b) the above optimal design problem $(\widetilde{R P})$ admits optimal solutions;

(c) these solutions (in the sense of Young measures) show that optimal microstructures are first order laminates with normal $n=(0,1)$ and volume fraction $s$.

One can pass from $(R P)$ to $(\widetilde{R P})$ simply by minimizing the general relaxed integrand $\varphi(t, x, F, s)$ over the auxiliary variable $F^{(2)}$, the second row of $F$, keeping all other variables fixed (and taking $a_{\alpha}=1, a_{\beta}=1$ ). This is an elementary calculus exercise (Section 6). This vector, the second row of $F$, was introduced as an auxiliary field to go from the original formulation $(P)$ to its variational form $(V P)$. After relaxation, in which this auxiliary vector plays an important role, we eliminate it by minimizing over it, so that we are back to a state law which is the result of this minimization process. More importantly, first order laminates involved in this process (the passage from $(R P)$ to $(\widetilde{R P}))$ always correspond to normal direction $n=(0,1)$, i.e., the optimal laminates have to be arranged in the direction perpendicular to the space axis with volume fraction $s(t, x)$. These are, in particular, laminates of the class considered by Lurie in the previously cited works. Our conjecture is that this procedure should capture the optimal relaxed state law.

Notice how this process cannot produce a general relaxation theorem (this is in fact our previous theorem for $(R P)$ ), as it is tailored and computed for the particular choice of the coefficients $a_{\alpha}=1$, $a_{\beta}=1$. It asserts that among the many relaxed wave-like equations that can be produced by mixing dynamically the two materials, the one providing optimal microstructures for the particular choice of the coefficients $a_{\alpha}=1, a_{\beta}=1$ is precisely the one obtained by replacing $\chi \in\{0,1\}$ by $s \in[0,1]$ (as in the situation of [27]). For other choices of the coefficients $a_{\alpha}$ and $a_{\beta}$, the optimal relaxed equation would possibly be different. One can see this phenomenon for the elliptic situation in [9]. The importance of having this more "economic" relaxation (compare Conjecture 1 with Theorem 1 p is that simulations can be performed for these, while it is out of the question to use $(R P)$ directly. We have written this in the form of a conjecture because, even though the passage from $(R P)$ to $(\widetilde{R P})$ is elementary, its formal rigorous proof requires a careful analysis. It has been shown to be correct in a number of situations in the elliptic case $([26])$. For our situation here, showing the validity of the conjecture is in progress $([18])$.

The paper is organized as follows. In Section 2, we describe in more detail the equivalent variational reformulation as well as a general relaxation result when integrands are not continuous and may take on infinite values abruptly. As there is nothing new here compared to other previous works in the elliptic case, our description is rather a reminder included for the sake of completeness. Sections 3 and 4 are technical in nature but interesting, as we first compute a lower bound of the constrained quasiconvexification (Section 3), by using in a fundamental way the weak continuity of the determinant. Section 4 is concerned with the search for laminates furnishing the precise value of the lower bound in an attempt to show equality of the three convex hulls (poly-, quasi- and rankone convex hulls), as is standard in this kind of calculation. In Section 5, we show some particular examples of this relaxation for different and interesting choices of the coefficients $a_{\alpha}, a_{\beta}$. Finally, in Section 6 we analyze the relaxed problem and propose a simpler relaxation, while in Section 7 we numerically solve it by using a gradient descent method.

\section{Reformulation and relaxation}

The lack of classical solution of the original optimal design problem is well-established. We propose to reformulate the problem as a vector variational problem to which we apply suitable tools to study its relaxation. We follow a similar approach to the one in [2, 23]. 
Under the hypothesis of simple connectedness of $\Omega$ (an interval), there exists a potential $v \in$ $H^{1}((0, T) \times \Omega)$ such that the state equation can be recast as

$$
-\operatorname{div}\left(u_{t}(t, x),-[\alpha \chi(t, x)+\beta(1-\chi(t, x))] u_{x}(t, x)\right)=0 \quad \text { in }[0, T] \times \Omega,
$$

where the div operator is now considered with respect to the variables $t$ and $x$. The state equation is equivalent to the pointwise constraint

$$
\left(\begin{array}{c}
u_{t}(t, x) \\
-[\alpha \chi(t, x)+\beta(1-\chi(t, x))] u_{x}(t, x)
\end{array}\right)=R \nabla v(t, x) \quad \text { a.e. }(t, x) \in[0, T] \times \Omega,
$$

where $R$ is the counterclockwise $\pi / 2$-rotation in the space-time plane. If we let $\Lambda_{-\gamma}$ be as in 3 , this constraint reads

$$
\left(\begin{array}{c}
\nabla u(t, x) \\
\nabla v(t, x)
\end{array}\right) \in \Lambda_{-\alpha} \cup \Lambda_{-\beta} \quad \text { a.e. }(t, x) \in[0, T] \times \Omega .
$$

It is clear that we can identify the design variable $\chi$ with the vector field $U=(u, v)$ complying with (4); and conversely, a pair $U=(u, v)$ which satisfies (4) determines a characteristic function $\chi$, so that we can consider the new design variable $U=\left(U^{(T)}, U^{(2)}\right)=(u, v)$, where $U: \mathbb{R}^{2} \rightarrow \mathbb{R}^{2}$ and $\nabla U(t, x) \in \mathbb{R}^{2 \times 2}$, under the main constraint 4 .

Therefore, by using the above statement and the notation of the Introduction, it is easy to check that the original optimal design problem $(P)$ is equivalent to the variational problem $(V P)$.

We have thus recast our optimal design problem as a typical variational problem. We see that it is a non-convex vector problem, which we are going to analyze by seeking its relaxation. We use Young measures as the main tool in the computation of a suitable density for the relaxed problem. In this sense, we can rely on the following relaxation result [2] whose main idea has been a useful tool in other situations [2, 23, 25].

We denote the initial condition (1) by I.C., the boundary condition (2) by B.C. and put

$$
\begin{aligned}
m=\inf \left\{\int_{\Omega} \int_{0}^{T} W(t, x, \nabla U(t, x)) \mathrm{d} t \mathrm{~d} x\right. & \\
U \in H^{1}((0, T) \times \Omega)^{2}, & U^{(1)} \text { satisfies the B.C. and I.C., } \\
& \left.\int_{\Omega} V(t, x, \nabla U(t, x)) \mathrm{d} x \leqslant V_{\alpha}|\Omega|, \forall t \in[0, T]\right\} .
\end{aligned}
$$

We know [2] that

$$
\begin{aligned}
& m \geqslant \bar{m}=\inf \left\{\int_{\Omega} \int_{0}^{T} C Q W(t, x, \nabla U(t, x), s(t, x)) \mathrm{d} t \mathrm{~d} x:\right. \\
& U \in H^{1}([0, T] \times \Omega)^{2}, U^{(1)} \text { satisfies the B.C. and I.C., } \\
& \left.0 \leqslant s(t, x) \leqslant 1, \int_{\Omega} s(t, x) \mathrm{d} x \leqslant V_{\alpha}|\Omega|, \forall t \in[0, T]\right\},
\end{aligned}
$$

where

$$
C Q W(t, x, F, s)=\inf \left\{\int_{M^{2 \times 2}} W(t, x, A) \mathrm{d} v(A): v \in \mathcal{A}(F, s)\right\}
$$


with

$$
\begin{aligned}
& \mathcal{A}(F, s)=\left\{v: v \text { is a homogeneous } H^{1}\right. \text {-Young measure, } \\
& \left.\qquad F=\int_{M^{2 \times 2}} A \mathrm{~d} v(A), \int_{M^{2 \times 2}} V(t, x, A) \mathrm{d} \nu(A)=s\right\} .
\end{aligned}
$$

Notice that the inequality $m \geqslant \bar{m}$ will be an equality when $W$ is a Carathéodory function with appropriate growth constrains. However, in our situation it is still possible to prove this equality despite the fact that $W$ is not a Carathéodory function. Let us consider the minimization problem

$$
\tilde{m}=\inf \left\{\int_{\Omega} \int_{0}^{T} \int_{M^{2 \times 2}} W(t, x, A) \mathrm{d} v_{t, x}(A) \mathrm{d} t \mathrm{~d} x: v \in \mathcal{B}\left(\text { B.C., I.C., } V_{\alpha}\right)\right\},
$$

where

$$
\begin{aligned}
& \mathcal{B}\left(\text { B.C., I.C., } V_{\alpha}\right)=\left\{v: H^{1} \text {-Young meas., } \operatorname{supp}\left(v_{t, x}\right) \subset \Lambda_{\alpha} \cup \Lambda_{\beta}\right. \text {, } \\
& \exists U \in H^{1}([0, T] \times \Omega)^{2}, U^{(1)} \text { satisfies the I.C. and B.C., } \\
& \nabla U(t, x)=\int_{M^{2 \times 2}} A \mathrm{~d} v_{t, x}(A) \\
& \left.\int_{\Omega} \int_{M^{2 \times 2}} V(t, x, A) \mathrm{d} v_{t, x}(A) \mathrm{d} x \leqslant V_{\alpha}|\Omega|, \forall t \in[0, T]\right\} .
\end{aligned}
$$

We have the following result, whose proof is essentially identical to the one in [2].

THEOREM 3 ([2]) Suppose that the initial data $u_{0}$ and $u_{1}$ have the regularity

$$
u_{0} \in H^{2}(0,1) \cap H_{0}^{1}(0,1), \quad u_{1} \in H_{0}^{1}(0,1) .
$$

Then

$$
m=\bar{m}=\tilde{m}
$$

Moreover, for each measure $v \in \mathcal{B}$ (B.C., I.C., $\left.V_{\alpha}\right)$ such that $\operatorname{supp}\left(v_{x, t}\right) \subset \Lambda_{\alpha} \cup \Lambda_{\beta}$ for a.e. $(t, x) \in$ $[0, T] \times \Omega$, there exists a sequence $\left\{\nabla U_{k}\right\}$ such that

(i) $U_{k} \in\left(H^{1}([0, T] \times \Omega)\right)^{2}, U^{(1)}$ satisfies the I.C. and B.C., and $\left\{\left|\nabla U_{k}\right|^{2}\right\}$ is equi-integrable,

(ii) $\nabla U_{k}(t, x) \in \Lambda_{\alpha} \cup \Lambda_{\beta}$ for a.e. $(t, x) \in[0, T] \times \Omega$ and all $k$, and

$$
\int_{\Omega} V\left(t, x, \nabla U_{k}(t, x)\right) \mathrm{d} x \leqslant V_{\alpha}|\Omega|, \quad \forall t \in[0, T] \forall k
$$

$$
\lim _{k \rightarrow \infty} \int_{0}^{T} \int_{\Omega} W\left(t, x, \nabla U_{k}(t, x)\right) \mathrm{d} x \mathrm{~d} t=\int_{0}^{T} \int_{\Omega} \int_{M^{2 \times 2}} W(t, x, A) \mathrm{d} v_{t, x}(A) \mathrm{d} x \mathrm{~d} t .
$$

The only remark worth making concerns the regularity of initial data. The proof of this theorem in the elliptic case in [2] relies in a fundamental way on the elliptic character of the manifolds $\Lambda_{\gamma}$ to discard concentrations of the sequence $\left\{\left|\nabla U_{k}\right|^{2}\right\}$. For the hyperbolic case, this equi-integrability can also be shown, in a standard way, based on the regularity of solutions for the wave equation (with uniformly elliptic spatial part) coming from the regularity of initial conditions (see [13]). 


\section{The lower bound: polyconvexification}

We would like to compute explicitly the constrained quasiconvexification defined as

$$
C Q W(t, x, F, s)=\inf \left\{\int_{M^{2 \times 2}} W(t, x, A) \mathrm{d} v(A): v \in \mathcal{A}(F, s)\right\}
$$

where $\mathcal{A}(F, s)$ is given in (5). Since the variable $(t, x) \in[0, T] \times \Omega$ can be considered as a parameter, we drop this dependence to simplify the notation. In this form, the constrained quasiconvexification can be expressed as

$$
\inf _{\nu}\left\{\int_{M^{2 \times 2}} W(A) \mathrm{d} v(A): F=\int_{M^{2 \times 2}} A \mathrm{~d} v(A), \int_{M^{2 \times 2}} V(A) \mathrm{d} \nu(A)=s, \forall t \in[0, T]\right\}
$$

with $v$ a homogeneous $H^{1}$-Young measure with $\operatorname{supp}(v) \subset \Lambda_{\alpha} \cup \Lambda_{\beta}$.

For $(F, s)$ (and $(t, x))$ fixed, we are going to compute the value of (6), i.e. $C Q W(t, x, F, s)$. The main difficulty here is that we do not know explicitly the set of admissible measures, which we denote as $\mathcal{A}$. We propose the following strategy. Consider two classes $\mathcal{A}_{*}, \mathcal{A}^{*}$ of probability measures such that

$$
\mathcal{A}_{*} \subset \mathcal{A} \subset \mathcal{A}^{*} \text {. }
$$

We first calculate the minimum over the greater class $\mathcal{A}^{*}$, and then we check that the optimal value is attained by at least one measure over the narrower class $\mathcal{A}_{*}$. This tells us that the optimal value so achieved is the same in $\mathcal{A}$, and hence we will have in fact computed the exact value $C Q W(t, x, F, s)$.

Following [23], we choose for $\mathcal{A}^{*}$ the set of polyconvex measures, which are not necessarily gradient Young measures, and therefore obtain a lower bound (the (constrained) polyconvexification). The main property of these measures is that they commute with the determinant. This constraint can be imposed in a more or less manageable way. We also choose for $\mathcal{A}_{*}$ the class of laminates, which is a subclass of the gradient Young measures. By working with this class, we would get an upper bound (the (constrained) rank-one convexification).

The polyconvexification $C P W(F, s)$ can be computed through the optimization problem

$$
\min _{v} \int_{M^{2 \times 2}} W(A) \mathrm{d} v(A)
$$

where

$$
v \in \mathcal{A}(F, s)=\{v: v \text { is a homogeneous Young measure }
$$

$$
\begin{aligned}
& \text { which commutes with det, } F=\int_{M^{2 \times 2}} A \mathrm{~d} v(A), \\
& \left.\int_{M^{2 \times 2}} V(A) \mathrm{d} \nu(A)=s\right\} .
\end{aligned}
$$

From (8) we have the decomposition

$$
v=t v_{\alpha}+(1-t) v_{\beta}, \quad \operatorname{supp}\left(v_{\gamma}\right) \subset \Lambda_{\gamma}, \quad \gamma=\alpha, \beta,
$$

and therefore, from (7),

$$
F=s \int_{\Lambda_{\alpha}} A \mathrm{~d} v_{\alpha}(A)+(1-s) \int_{\Lambda_{\beta}} A \mathrm{~d} v_{\beta}(A) .
$$


If we put

$$
F_{\gamma}=\int_{\Lambda_{\gamma}} A \mathrm{~d} \nu_{\gamma}(A), \quad \gamma=\alpha, \beta,
$$

we have $F_{\gamma} \in \Lambda_{\gamma}$ for $\gamma=\alpha, \beta$, so from this property and (9), we have a non-compatible system on $F_{\gamma}$ unless

$$
F_{11}+F_{22}=0, \quad \text { i.e. } \operatorname{tr}(F)=0 .
$$

Let us suppose henceforth that this compatibility condition holds. This condition lets us simplify the problem from $2 \times 2$ matrices to 3 -d vectors, using the identification

$$
F=\left(\begin{array}{cc}
x & y \\
z & -x
\end{array}\right) \leftrightarrow(x, y, z)
$$

Therefore the manifolds $\Lambda_{\gamma}$ can be rewritten as

$$
\Lambda_{\gamma}=\left\{(\boldsymbol{x}, \boldsymbol{y}, \boldsymbol{z}) \in \mathbb{R}^{3}: \boldsymbol{z}+\gamma \boldsymbol{y}=0\right\} .
$$

In this way, the above system does not uniquely determine its solution. Indeed,

$$
F_{\alpha}=\left(\lambda, y_{\alpha},-\alpha y_{\alpha}\right), \quad F_{\beta}=\left(\frac{x-s \lambda}{1-s}, y_{\beta},-\beta y_{\beta}\right),
$$

where

$$
y_{\alpha}=\frac{1}{s(\beta-\alpha)}(\beta \boldsymbol{y}+\boldsymbol{z}), \quad y_{\beta}=\frac{-1}{(1-s)(\beta-\alpha)}(\alpha \boldsymbol{y}+\boldsymbol{z})
$$

and $\lambda \in \mathbb{R}$. We can check that if $A=\left(a_{1}, a_{2}, a_{3}\right) \in \Lambda_{\gamma}$ with $\gamma=\alpha, \beta$, then

$$
\operatorname{det} A=-a_{1}^{2}+\gamma a_{2}^{2},
$$

and by using the important constraint about the commutativity with det, we know that

$$
\begin{aligned}
\operatorname{det} F & =\int_{\mathbb{R}^{3}} \operatorname{det} A \mathrm{~d} v(A)=s \int_{\mathbb{R}^{3}} \operatorname{det} A \mathrm{~d} v_{\alpha}(A)+(1-s) \int_{\mathbb{R}^{3}} \operatorname{det} A \mathrm{~d} v_{\beta}(A) \\
& =-\int_{\mathbb{R}} a_{1}^{2} \mathrm{~d} v^{(1)}(A)+s \alpha \int_{\mathbb{R}^{2}} a_{2}^{2} \mathrm{~d} v_{\alpha}^{(2)}(A)+(1-s) \beta \int_{\mathbb{R}} a_{2}^{2} \mathrm{~d} v_{\beta}^{(2)}(A),
\end{aligned}
$$

where $v_{\gamma}^{i}$ designates the projection of $v_{\gamma}$ onto the $i$-th component.

On the other hand, we can write the cost functional in the form

$$
\int_{\mathbb{R}^{3}} W(A) \mathrm{d} v(A)=\int_{\mathbb{R}^{3}} a_{1}^{2} \mathrm{~d} v(A)+s a_{\alpha} \int_{\mathbb{R}^{3}} a_{2}^{2} \mathrm{~d} v_{\alpha}(A)+(1-s) a_{\beta} \int_{\mathbb{R}^{3}} a_{2}^{2} \mathrm{~d} v_{\beta}(A),
$$

so if we put

$$
S_{1}=\int_{\mathbb{R}^{3}} a_{1}^{2} \mathrm{~d} v(A), \quad S_{\gamma}=\int_{\Lambda_{\gamma}} a_{2}^{2} \mathrm{~d} \nu_{\gamma}(A) \quad \text { with } \gamma=\alpha, \beta,
$$

and use Jensen's inequality, we have the constraints

$$
S_{1} \geqslant x^{2}, \quad S_{\gamma} \geqslant y_{\gamma}^{2}, \quad \gamma=\alpha, \beta .
$$


By using the notation just introduced, the above inequalities and the constraint on the determinant, the constrained polyconvexification is given by the linear programming problem

$$
\underset{\left(S_{1}, S_{\gamma}, x_{\gamma}\right)}{\operatorname{minimize}} S_{1}+s a_{\alpha} S_{\alpha}+(1-s) a_{\beta} S_{\beta}
$$

subject to

$$
-\operatorname{det} F=S_{1}-s \alpha S_{\alpha}-(1-s) \beta S_{\beta}, \quad S_{1} \geqslant x^{2}, \quad S_{\gamma} \geqslant y_{\gamma}^{2} \quad \text { with } \gamma=\alpha, \beta \text {. }
$$

We can eliminate $S_{1}$ by replacing its value from the equality constraint in the cost functional. By so doing, only the variables $\left(S_{\alpha}, S_{\beta}\right)$ occur, with inequality constraints (see Figure 1 for a geometrical interpretation of the programming problem). It is easy to solve this problem. Under the conditions $a_{\alpha} \geqslant-\alpha$ and $a_{\beta} \geqslant-\beta$, the optimal value depends on the relative position of the oblique line and the $P$ point. Namely, the optimal solution can be attained at $P, P_{1}$ or $P_{2}$.

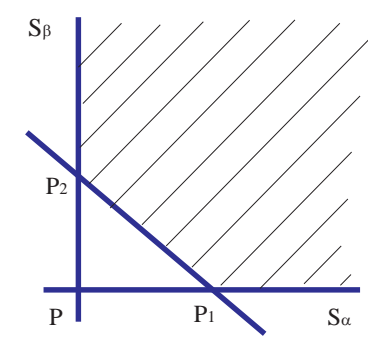

FIG. 1. New mathematical programming problem

If we define the function

$$
\psi(F, s)=\boldsymbol{y} \boldsymbol{z}+\frac{\alpha}{s(\beta-\alpha)^{2}}(\beta \boldsymbol{y}+\boldsymbol{z})^{2}+\frac{\beta}{(1-s)(\beta-\alpha)^{2}}(\alpha \boldsymbol{y}+\boldsymbol{z})^{2},
$$

the optimal value is

$$
\begin{cases}\frac{h}{s \beta(\beta-\alpha)^{2}}\left(\beta^{2} \boldsymbol{y}^{2}+z^{2}+2 \beta \boldsymbol{y} \boldsymbol{z}\right)+\boldsymbol{x}^{2}-\frac{a_{\beta}}{\beta} \boldsymbol{y} \boldsymbol{z} & \text { if } h(x, t) \geqslant 0, \psi(F, s) \leqslant 0 \\ \frac{-h}{(1-s) \alpha(\beta-\alpha)^{2}}\left(\alpha^{2} \boldsymbol{y}^{2}+\boldsymbol{z}^{2}+2 \alpha \boldsymbol{y} \boldsymbol{z}\right)+\boldsymbol{x}^{2}-\frac{a_{\alpha}}{\alpha} y z & \text { if } h(x, t) \leqslant 0, \psi(F, s) \leqslant 0 \\ -\operatorname{det} F+\frac{1}{s(1-s)(\beta-\alpha)^{2}}\left(\left((1-s) \beta^{2}\left(\alpha+a_{\alpha}\right)+s \alpha^{2}\left(\beta+a_{\beta}\right)\right) \boldsymbol{y}^{2}\right. & \\ \left.+\left((1-s)\left(\alpha+a_{\alpha}\right)+s\left(\beta+a_{\beta}\right)\right) z^{2}+2\left(\left(\alpha+a_{\alpha}\right) \beta-s \beta\right) \boldsymbol{y} z\right) & \text { if } \psi(F, s) \geqslant 0 .\end{cases}
$$

In addition, the optimal value is attained at

$$
\begin{array}{cl}
P_{1}: S_{\alpha}=y_{\alpha}^{2} \text { and } S_{1}=x^{2} & \text { if } h(x, t) \geqslant 0, \psi(F, s) \leqslant 0, \\
P_{2}: S_{\beta}=y_{\beta}^{2} \text { and } S_{1}=x^{2} & \text { if } h(x, t) \leqslant 0, \psi(F, s) \leqslant 0, \\
P: S_{\alpha}=y_{\alpha}^{2} \text { and } S_{\beta}=y_{\beta}^{2} & \text { if } \psi(F, s) \geqslant 0 .
\end{array}
$$


Therefore we have an explicit computation of the constrained polyconvexification given by

$$
C P W(F, s)=\left\{\begin{array}{cc}
\frac{h}{s \beta(\beta-\alpha)^{2}}\left(\beta^{2} y^{2}+z^{2}+2 \beta \boldsymbol{y} \boldsymbol{z}\right)+\boldsymbol{x}^{2}-\frac{a_{\beta}}{\beta} \boldsymbol{y} \boldsymbol{z} \\
\text { if } h(x, t) \geqslant 0, \psi(s, F) \leqslant 0, \operatorname{tr}(F)=0, \\
\frac{-h}{(1-s) \alpha(\beta-\alpha)^{2}}\left(\alpha^{2} \boldsymbol{y}^{2}+\boldsymbol{z}^{2}+2 \alpha \boldsymbol{y} \boldsymbol{z}\right)+\boldsymbol{x}^{2}-\frac{a_{\alpha}}{\alpha} \boldsymbol{y} \boldsymbol{z}, \\
\text { if } h(x, t) \leqslant 0, \psi(s, F) \leqslant 0, \operatorname{tr}(F)=0, \\
\frac{1}{s(1-s)(\beta-\alpha)^{2}}\left(\left((1-s) \beta^{2}\left(\alpha+a_{\alpha}\right)+s \alpha^{2}\left(\beta+a_{\beta}\right)\right) \boldsymbol{y}^{2}\right. \\
\left.+\left((1-s)\left(\alpha+a_{\alpha}\right)+s\left(\beta+a_{\beta}\right)\right) z^{2}+2\left(\left(\alpha+a_{\alpha}\right) \beta-s \beta\right) \boldsymbol{y} \boldsymbol{z}\right)-\operatorname{det} F \\
\text { if } \psi(s, F) \geqslant 0, \operatorname{tr}(F)=0, \\
\text { if } \operatorname{tr}(F) \neq 0 .
\end{array}\right.
$$

We claim that in fact this is an exact value. This amounts to finding laminates which yield this same optimal value.

\section{Optimal microstructures: laminates}

We have the lower bound given by the polyconvexification, and we will show that this bound is in fact attained. To this end, we seek an optimal microstructure (a laminate) whose second moments recover the value of the bound.

We try to find $v=s v_{\alpha}+(1-s) v_{\beta}$, a laminate with $\operatorname{supp}\left(v_{\gamma}\right) \subset \Lambda_{\gamma}, \gamma=\alpha, \beta, s \in(0,1)$, and first moment $F$. We have different optimal conditions depending on the sign of $\psi$ and $h$, and we analyze different cases accordingly.

\subsection{Case $\psi \geqslant 0$}

We start with the case when $\psi(F, s) \geqslant 0$ holds. In this case the optimal conditions (12) tell us that

$$
S_{\alpha, 2}=y_{\alpha}^{2}, \quad S_{\beta, 2}=y_{\beta}^{2}
$$

and therefore, by the strict convexity of the square function, we can deduce that

$$
v_{\gamma}^{(2)}=\delta_{y_{\gamma}}, \quad \gamma=\alpha, \beta .
$$

Hence

$$
F_{\alpha}=\left(\lambda, y_{\alpha},-\alpha y_{\alpha}\right), \quad F_{\beta}=\left(\frac{x-s \lambda}{1-s}, y_{\beta},-\beta y_{\beta}\right),
$$

with $\lambda \in \mathbb{R}$ arbitrary. This means that for every $\lambda \in \mathbb{R}$ we can decompose $F$ as a convex combination of two matrices in $\Lambda_{\alpha}, \Lambda_{\beta}$ respectively, and satisfying the volume constraint (see Figure 22.

The next step is to check that there exist some $\lambda \in \mathbb{R}$ such that $\operatorname{rank}\left(F_{\alpha}-F_{\beta}\right)=1$. After some algebra, we can write

$$
\operatorname{rank}\left(F_{\alpha}-F_{\beta}\right)=1 \Leftrightarrow C_{F, s}(\lambda)=0
$$




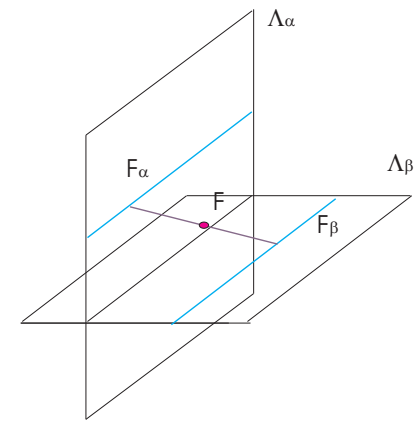

FIG. 2. Infinite decompositions of $F$

where

$$
C_{F, s}(\lambda)=-\operatorname{det} F-s\left(\lambda^{2}-\alpha y_{\alpha}^{2}\right)-(1-s)\left(\left(\frac{F_{11}-s \lambda}{1-s}\right)^{2}-\beta y_{\beta}^{2}\right)
$$

is a second degree polynomial on $\lambda$. It is easy to check that the discriminant of $C_{F, s}$ is $\psi(F, s)$, and so its roots are

$$
\lambda_{i}=x+(-1)^{i} \sqrt{\frac{1-s}{s} \psi(F, s)}, \quad i=1,2 .
$$

Therefore for all pairs $(F, s)$ such that $\psi(F, s) \geqslant 0$, there exist two first order laminates

$$
v=s \delta_{F_{\alpha, i}}+(1-s) \delta_{F_{\beta, i}}, \quad i=1,2,
$$

where

$$
F_{\alpha, i}=\left(\begin{array}{cc}
\lambda_{i} & F_{\alpha, 12} \\
-\alpha F_{\alpha, 12} & -\lambda_{i}
\end{array}\right), \quad F_{\beta, i}=\left(\begin{array}{cc}
\frac{x-s \lambda_{i}}{1-s} & F_{\beta, 12} \\
-\beta F_{\beta, 12} & -\frac{x-s \lambda_{i}}{1-s}
\end{array}\right)
$$

and they provide the optimal value of the polyconvexification.

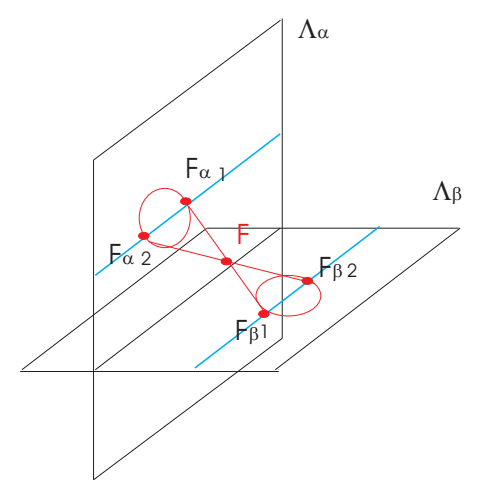

FIG. 3. Two first order laminates

Thanks to the spatial identification $F=(\boldsymbol{x}, \boldsymbol{y}, \boldsymbol{z})$, we can observe the above computations from a geometric point of view (see Figure 3). For any matrix $F=(\boldsymbol{x}, \boldsymbol{y}, \boldsymbol{z})$ the determinant is 
det $F=-\left(\boldsymbol{x}^{2}+\boldsymbol{y} \boldsymbol{z}\right)$, which means that for any matrix $F$ there exists a cone $\left\{\boldsymbol{x}^{2}+\boldsymbol{y} \boldsymbol{z}=0\right\}$ of rankone directions through this matrix. From optimality conditions we obtain an explicit identification (13) of $F_{\gamma}, \gamma=\alpha, \beta$, up to the first component, which gives us a degree of freedom in the search of the optimal decomposition. Geometrically, we notice that the intersection between the manifolds $\Lambda_{\gamma}$ and the rank-one cone are ellipses, whose intersection with the admissible $F_{\gamma}$ are two points $F_{\gamma, i}, \gamma=\alpha, \beta$ and $i=1,2$.

\subsection{Case $\psi \leqslant 0$}

We now study the other case, $\psi(F, s) \leqslant 0$. In this situation, we have two different optimal conditions depending on the sign of $h$. We treat the case $h \geqslant 0$. The other case is similar.

From the optimal condition (10) for this case, we have

$$
S_{\alpha, 2}=y_{\alpha}^{2}, \quad S_{1}=x^{2},
$$

and by using similar arguments to those above, we can deduce

$$
v_{\alpha}^{(2)}=\delta_{y_{\alpha}}, \quad v^{(1)}=\delta_{x},
$$

where

1. $v_{\alpha}=\delta_{F_{\alpha}}$ with

$$
F_{\alpha}=\left(\boldsymbol{x}, y_{\alpha},-\alpha y_{\alpha}\right),
$$

2. since $F$ is the first moment of $v$, there exists a unique decomposition

$$
F=s F_{\alpha}+(1-s) F_{\beta}
$$

with $F_{\gamma} \in \Lambda_{\gamma}, \gamma=\alpha, \beta$, where $F_{\alpha}$ is of the form just indicated, and

$$
F_{\beta}=\left(\boldsymbol{x}, y_{\beta},-\beta y_{\beta}\right) .
$$

Consider a pair $(F, s)$ such that $\psi(F, s)<0$. After an elementary manipulation, we get

$$
\begin{aligned}
\psi(F, s) \leqslant 0 \Leftrightarrow & -(\beta-\alpha)^{2} \boldsymbol{y} z s^{2}+\left(\alpha \beta(\alpha-\beta) \boldsymbol{y}^{2}+(\beta-\alpha) \boldsymbol{z}^{2}+(\beta-\alpha)^{2} \boldsymbol{y} \boldsymbol{z}\right) s \\
& +\left(\alpha \beta^{2} \boldsymbol{y}^{2}+\alpha \boldsymbol{z}^{2}+2 \alpha \beta \boldsymbol{y} \boldsymbol{z}\right) \leqslant 0 .
\end{aligned}
$$

Let $P_{F}(s)$ be this second degree polynomial in $s$ for fixed $F$. The set where $\psi(F, s) \leqslant 0$ is the set where $P_{F}$ has solutions in $[0,1]$, and $s$ lies between those two solutions. There exist real solutions if the discriminant is non-negative, and, in addition, it is easy to check that $P_{F}(0), P_{F}(1)$ are positive ${ }^{1}$ if $F \notin \Lambda_{\alpha} \cup \Lambda_{\beta}$. Therefore there are positive solutions if $P_{F}$ is decreasing at 0 .

After some algebra the discriminant is

$$
g(F)=\alpha^{2} \beta^{2} \boldsymbol{y}^{4}+\boldsymbol{z}^{4}+\left(\alpha^{2}+4 \beta \alpha+\beta^{2}\right) \boldsymbol{y} z+2 \alpha \beta \boldsymbol{y}^{3} \boldsymbol{z}+2(\alpha+\beta) \boldsymbol{z}^{3} \boldsymbol{y} \geqslant 0
$$

and the decreasing condition is

$$
h(F)=(\alpha+\beta) \boldsymbol{y} z+\alpha \beta \boldsymbol{y}^{2}+z^{2} \leqslant 0 .
$$

${ }^{1} P_{F}(0)=\alpha|\beta \boldsymbol{y}+\boldsymbol{z}|^{2}, P_{F}(1)=\beta|\alpha \boldsymbol{y}+\boldsymbol{z}|^{2}$. 
Therefore the set of pairs $(F, s)$ where $\psi(F, s) \leqslant 0$ can be described as

$$
\left\{(F, s) \in M^{2 \times 2} \times \mathbb{R}: g(F) \geqslant 0, h(F) \leqslant 0, s \in\left(r_{1}, r_{2}\right)\right\},
$$

where

$$
r_{i}=\frac{1}{2}-\frac{1}{2(\beta-\alpha) \boldsymbol{y} z}\left(\alpha \beta \boldsymbol{y}^{2}-z^{2}+(-1)^{i} \sqrt{g(F)}\right), \quad i=1,2 .
$$

We thus have a characterization of the set $\psi(F, s) \leqslant 0$. We now look for rank-one connections between both manifolds.

We would like to write

$$
F=r B_{\alpha}+(1-r) B_{\beta}
$$

with $r \in(0,1), B_{\gamma} \in \Lambda_{\gamma},\left(B_{\gamma}\right)_{1}=x, \gamma=\alpha, \beta$, and $\operatorname{rank}\left(B_{\alpha}-B_{\beta}\right)=1$.

On the one hand,

$$
\left.\begin{array}{l}
B_{\gamma} \in \Lambda_{\gamma} \\
\left(B_{\gamma}\right)_{1}=x
\end{array}\right\} \Rightarrow B_{\gamma}=\left(x, y_{\gamma},-\gamma y_{\gamma}\right), \quad \gamma=\alpha, \beta .
$$

The constraint on the vanishing determinant can be rewritten, after some manipulation, as

$$
P_{F}(r)=0,
$$

whose roots are $r_{i}$. We can therefore guarantee that there exist two rank-one directions between $\Lambda_{\alpha}$ and $\Lambda_{\beta}$ with barycenter $F$.

We are now in a position to find an optimal second order laminate which recovers the lower bound given by the polyconvexification. We take $v_{\alpha}=\delta_{F \alpha}$ and $v_{\beta}$ as a convex combination of two Dirac masses supported in the $\beta$ manifold (see Figure 4 ).

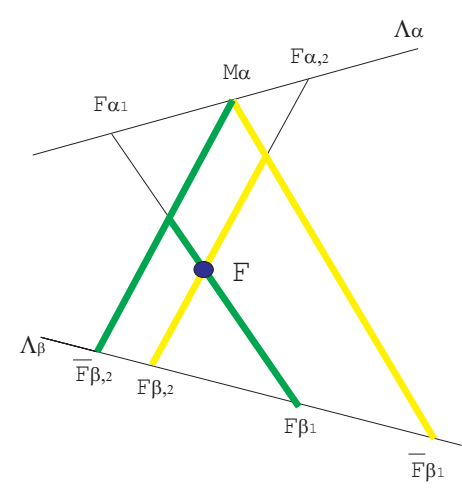

FIG. 4. Second order laminates

Put

$$
F_{\beta, i}=\left(\boldsymbol{x}, y_{\beta, i},-\beta y_{\beta, i}\right)
$$

with

$$
y_{\beta, i}=\frac{-1}{\left(1-r_{i}\right)(\beta-\alpha)}(\alpha \boldsymbol{y}+\boldsymbol{z}), \quad i=1,2 .
$$

Since $r_{1} \leqslant s \leqslant r_{2}$, it is clear that $y_{\beta}$ is a convex combination of $F_{\beta, i}, i=1,2$. 
If we consider $\bar{F}_{\beta, i}=F_{\alpha}+l_{i}\left(F_{\beta, i}-F_{\alpha, i}\right)$ with $l_{i}$ such that $\bar{F}_{\beta, i} \in \Lambda_{\beta}$, and take

$$
l_{i}=\frac{r_{i}}{s}, \quad \rho_{i, j}=\frac{\left(1-r_{j}\right)\left(r_{i}-s\right)}{r_{i}-r_{j}}, \quad \tau_{i, j}=\frac{\left(r_{j}-s\right)\left(r_{i}-1\right)}{r_{j}\left(1+r_{i}\right)+s\left(1-r_{j}\right)},
$$

we can define the second order laminate with support on $\Lambda_{\alpha} \cup \Lambda_{\beta}$, barycenter $F$, and mass in $\Lambda_{\alpha}$ equal to $s$, by putting

$$
v_{i, j}=\tau_{i, j} \delta_{F_{\beta, i}}+\left(1-\tau_{i, j}\right)\left(\rho_{i, j} \delta_{\bar{F}_{\beta, j}}+\left(1-\rho_{i, j}\right) \delta_{F_{\alpha}}\right)
$$

with $i, j \in\{1,2\}, i \neq j$, where

$$
\operatorname{det}\left(\bar{F}_{\beta, j}-F_{\alpha}\right)=0, \quad \operatorname{det}\left(F_{\beta, i}-\left(\rho_{i, j} \bar{F}_{\beta, j}+\left(1-\rho_{i, j}\right) F_{\alpha}\right)\right)=0 .
$$

Again, using the spatial identification we can interpret geometrically the above analytical computations. We lost the degree of freedom of the first component of the matrices $F_{\alpha}$ and $F_{\beta}$, since these matrices are explicitly determined by (14) and (15), and their first component is $\boldsymbol{x}$ in both cases. This fact lets us simplify the spatial situation to a 2-d case in the plane determined by the first component equal to $\boldsymbol{x}$. The intersection of the manifolds $\Lambda_{\gamma}$ and the cone of rank-one directions through $F$ reduces to two matrices in each manifold, which we have denoted $F_{\gamma, i}$. From these matrices connected by rank-one directions we can obtain a second order laminate with volume fraction $s$ on $\Lambda_{\alpha}$ and $1-s$ on $\Lambda_{\beta}$. This construction is shown in Figure 4 , where the spatial situation is reduced to the plane of the first component equal to $x$. A similar result holds for the other point where the optimal value is attained $(h(x, s) \leqslant 0)$.

We summarize all of these computations of optimal laminates leading to the relaxed integrand $\varphi$.

When $\psi(F, s) \geqslant 0$ there exist two optimal first order laminates leading to the value of the relaxed integrand $\varphi$,

$$
v=s \delta_{F_{\alpha, i}}+(1-s) \delta_{F_{\beta, i}}, \quad i=1,2,
$$

where

$$
F_{\alpha, i}=\left(\begin{array}{cc}
\lambda_{i} & F_{\alpha, 12} \\
-\alpha F_{\alpha, 12} & -\lambda_{i}
\end{array}\right), \quad F_{\beta, i}=\left(\begin{array}{cc}
\frac{F_{11}-s \lambda_{i}}{1-s} & F_{\beta, 12} \\
-\beta F_{\beta, 12} & -\frac{F_{11}-s \lambda_{i}}{1-s}
\end{array}\right)
$$

with

$$
\begin{gathered}
\lambda_{i}=F_{11}+(-1) \sqrt[i]{\frac{1-s}{s} \psi(F, s)}, \quad i=1,2, \\
F_{\alpha, 12}=\frac{1}{s(\beta-\alpha)}\left(\beta F_{12}+F_{21}\right), \quad F_{\beta, 12}=\frac{-1}{(1-s)(\beta-\alpha)}\left(\alpha F_{12}+F_{21}\right) .
\end{gathered}
$$

When $\psi(F, s) \leqslant 0$ and $h(x, t) \geqslant 0$, there exist two optimal second order laminates

$$
v_{i, j}=\tau_{i, j} \delta_{F_{\beta, i}}+\left(1-\tau_{i, j}\right)\left(\rho_{i, j} \delta_{\bar{F}_{\beta, j}}+\left(1-\rho_{i, j}\right) \delta_{F_{\alpha}}\right)
$$

with $i, j \in\{1,2\}, i \neq j$, where the scalars are

$$
\rho_{i, j}=\frac{\left(1-r_{j}\right)\left(r_{i}-s\right)}{r_{i}-r_{j}}, \quad \tau_{i, j}=\frac{\left(r_{j}-s\right)\left(r_{i}-1\right)}{r_{j}\left(1+r_{i}\right)+s\left(1-r_{j}\right)}
$$


and the matrices are

$$
F_{\alpha}=\left(\begin{array}{cc}
F_{11} & F_{\alpha, 12} \\
-\alpha F_{\alpha, 12} & -F_{11}
\end{array}\right), \quad F_{\beta, i}=\left(\begin{array}{cc}
F_{11} & F_{\beta, 12, i} \\
-\beta F_{\beta, 12, i} & -F_{11}
\end{array}\right)
$$

with

$$
\begin{gathered}
F_{\beta, 12, i}=\frac{-1}{\left(1-r_{i}\right)(\beta-\alpha)}\left(\alpha F_{12}+F_{21}\right), \quad i=1,2, \\
r_{i}=\frac{1}{2}-\frac{1}{2(\beta-\alpha) F_{12} F_{21}}\left(\alpha \beta\left|F_{12}\right|^{2}-\left|F_{21}\right|^{2}+(-1)^{i} \sqrt{g(F)}\right), \quad i=1,2, \\
\bar{F}_{\beta, i}=F_{\alpha}+l_{i}\left(F_{\beta, i}-F_{\alpha, i}\right), \quad l_{i}=\frac{r_{i}}{s} .
\end{gathered}
$$

Similarly, when $\psi(F, s) \leqslant 0$ and $h(x, t) \leqslant 0$, the optimal microstructure is another second order laminate given by

$$
v_{i, j}=\tau_{i, j} \delta_{F_{\alpha, i}}+\left(1-\tau_{i, j}\right)\left(\rho_{i, j} \delta_{\bar{F}_{\alpha, j}}+\left(1-\rho_{i, j}\right) \delta_{F_{\beta}}\right)
$$

with $i, j \in\{1,2\}, i \neq j$, where the scalars are

$$
\rho_{i, j}=\frac{r_{j}\left(r_{i}-s\right)}{r_{i}-r_{j}}, \quad \tau_{i, j}=\frac{\left(s-r_{j}\right) r_{i}}{r_{i}\left(r_{j}-1\right)+r_{j}(1-s)},
$$

and the matrices involved are

$$
F_{\beta}=\left(\begin{array}{cc}
F_{11} & F_{\beta, 12} \\
-\beta F_{\beta, 12} & -F_{11}
\end{array}\right), \quad F_{\alpha, i}=\left(\begin{array}{cc}
F_{11} & F_{\alpha, 12, i} \\
-\beta F_{\alpha, 12, i} & -F_{11}
\end{array}\right)
$$

with

$$
\begin{aligned}
F_{\alpha, 12, i} & =\frac{1}{r_{i}(\beta-\alpha)}\left(\beta F_{12}+F_{21}\right), \quad i=1,2, \\
\bar{F}_{\alpha, i} & =F_{\beta}-l_{i}\left(F_{\beta, i}-F_{\alpha, i}\right), \quad l_{i}=\frac{1-r_{i}}{1-s} .
\end{aligned}
$$

\section{Some particular examples}

In this section we exhibit some particular examples where, by using Theorem 1, we can compute the relaxed cost functional explicitly.

EXAMPLE 1 An interesting and academic example corresponds to $a_{\alpha}(t, x)=\alpha, a_{\beta}(t, x)=\beta$ so that $h \equiv 0$, the cost functional can be written as

$$
\int_{0}^{T} \int_{\Omega}\left[u_{t}^{2}(t, x)+(\alpha \chi+\beta(1-\chi)) u_{x}^{2}(t, x)\right] \mathrm{d} x \mathrm{~d} t,
$$

and the constrained quasiconvexification is

$$
\varphi(F, s)= \begin{cases}F_{11}^{2}-F_{12} F_{21} & \text { if } \psi(s, F) \leqslant 0, \\ -\operatorname{det} F+\frac{1}{s(1-s)(\beta-\alpha)^{2}}\left(2 \alpha \beta(s \alpha+(1-s) \beta)\left|F_{12}\right|^{2}\right. & \\ \left.+2((1-s) \alpha+s \beta)\left|F_{21}\right|^{2}+4 \alpha \beta F_{12} F_{21}\right) & \text { if } \psi(s, F) \geqslant 0 .\end{cases}
$$


EXAMPLE 2 Another interesting case occurs when we take $a(t, x, \chi)=1$, the simplest quadratic cost function but very interesting from the mathematical point of view. In this case the relaxed cost functional is

$$
\int_{0}^{T} \int_{\Omega}\left[u_{t}^{2}(t, x)+u_{x}^{2}(t, x)\right] \mathrm{d} x \mathrm{~d} t,
$$

and therefore $a_{\alpha}(t, x)=a_{\beta}(t, x)=1$. Hence

$$
h(t, x)=\beta-\alpha,
$$

and the constrained quasiconvexification simplifies to

$$
\varphi(F, s)=\left\{\begin{array}{rr}
\frac{1}{s \beta(\beta-\alpha)}\left(s \beta(\beta-\alpha)\left|F_{11}\right|^{2}+\beta^{2}\left|F_{12}\right|^{2}+\left|F_{21}\right|^{2}+(s \alpha+\beta(2-s)) F_{12} F_{21}\right) \\
-\operatorname{det} \psi(s, F) \leqslant 0, \\
\left.+((1-s) \alpha+s \beta+1)\left|F_{21}\right|^{2}+2(\beta(1-s)+\alpha(s+\beta)) F_{12} F_{21}\right) \\
\text { if } \psi(s, F) \geqslant 0 .
\end{array}\right.
$$

EXAMPLE 3 The last case lies on the border line for our computations to be valid. We take $a_{\alpha}(t, x)=-\alpha$ and $a_{\beta}(t, x)=-\beta$ so that $h$ identically vanishes. The cost functional is

$$
\int_{0}^{T} \int_{\Omega}\left[u_{t}^{2}(t, x)-(\alpha \chi+\beta(1-\chi)) u_{x}^{2}(t, x)\right] \mathrm{d} x \mathrm{~d} t,
$$

and for this case the relaxed integrand surprisingly is - det (recall the restriction on the trace):

$$
\varphi(F, s)=F_{11}^{2}+F_{12} F_{21}=-\operatorname{det} F .
$$

Note that depending on the choice of the coefficients $a_{\alpha}, a_{\beta}$ we obtain different cost densities for the relaxed problem, yet this choice is independent of the state equation. It is interesting to remark that for all these examples the optimal laminates correspond to the ones computed in the last section: (17) when $\psi \geqslant 0$ and $\left[18\right.$ when $\psi \leqslant 0$, which are independent of $a_{\alpha}, a_{\beta}$.

\section{Analysis of $(R P)$ in the quadratic case}

In this section we analyze the quadratic case which is Example 2 in the preceding section, and thus focus on $(R P)$ where the cost density is given by $[19)$.

From the previous sections we know that this problem admits optimal solutions, and moreover they are first or second order laminates depending on the sign of the function $\psi$. An interesting fact is that all functions involved are quadratic in the vector gradient variable and therefore regular, yet it is the presence of gradients and the pointwise constraint that make the problem difficult to examine.

One first attempt would lead us to look at optimality conditions introducing several multipliers to keep track of the restrictions. This makes the problem more difficult in the sense that we have to solve a system of partial differential equations. Instead we follow a similar strategy to [9]. The pointwise constraint given by $\psi$ depends only on the variables $F_{12}, F_{21}$, therefore we try to find the "optimal" relationship between these two variables. The next lemma is completely elementary. 
LEMMA 1 For fixed s, the optimal solution of the quadratic, mathematical programming problem

$$
\text { Minimize in } F_{(21)}: \varphi(F, s)
$$

occurs when

$$
(\alpha s+\beta(1-s)) F_{12}+F_{21}=0 .
$$

In addition, the associated optimal microstructures are first order laminates with volume fraction $s$ for the $\alpha$-material and orientation of layers always vertical (along the time axis):

$$
s(t, x) \delta_{F_{\alpha}}+(1-s(t, x)) \delta_{F_{\beta}}
$$

with normal direction of lamination $n=(0,1)$. Having in mind the trace condition $F_{11}+F_{22}=0$ the optimal value of the cost function simplifies to

$$
F_{11}^{2}+F_{12}^{2} \text {. }
$$

The idea is then to replace the complicated cost function $\varphi$ by the expression 20 and then minimize under the constraints

$$
(\alpha s+\beta(1-s)) F_{12}(t, x)+F_{21}(t, x)=0, \quad F_{11}(t, x)+F_{22}(t, x)=0,
$$

i.e.

$$
\left(\begin{array}{c}
F_{11}(t, x) \\
-[\alpha s(t, x)+\beta(1-s(t, x))] F_{12}(t, x)
\end{array}\right)=T F^{(2)}(x, t) \quad \text { a.e. }(t, x) \in[0, T] \times \Omega,
$$

which is equivalent to

$$
\operatorname{div}\left(\begin{array}{c}
F_{11}(t, x) \\
-[\alpha s(t, x)+\beta(1-s(t, x))] F_{12}(t, x)
\end{array}\right)=0 .
$$

Therefore we can write the minimization problem in terms of the original variable $U^{(1)}=u$ leading to the new relaxed problem (stated in Conjecture 1):

$$
(\widetilde{R P}) \quad \min _{s} \int_{0}^{T} \int_{\Omega}\left[u_{t}^{2}(t, x)+u_{x}^{2}(t, x)\right] \mathrm{d} x \mathrm{~d} t,
$$

where $u$ is the unique solution of

$$
\begin{array}{ll}
u_{t t}-\operatorname{div}\left([\alpha s+\beta(1-s)] u_{x}\right)=0 & \text { in }(0, T) \times(0,1), \\
u(0, x)=u_{0}(x), \quad u_{t}(0, x)=u_{1}(x) & \text { in } \Omega, \\
u(t, 0)=0, \quad u(t, 1)=0 & \text { in }[0, T] .
\end{array}
$$

This new problem may be seen as the continuous version of the original design problem in which the function $\chi(x, t)$ is replaced by the continuous function $s(x, t)$. We cannot prove directly that the above problem admits optimal solutions, though we claim, by our conjecture, that it indeed does because of the particular form of the problem and not as a consequence of general results. A deeper and exhaustive analysis of this problem is still in progress (see [18]). Hopefully, the existence of solutions of these problems will be proved. We support our conclusion numerically in the next section. All we can say at this point is contained in the following assertion. 
LEMMA 2 We have

$$
\inf (P)=\inf (\widetilde{R P})=\min (R P)
$$

Proof. It is easy to see that

$$
\inf (P) \geqslant \inf (\widetilde{R P}) \quad \text { and } \quad \inf (\widetilde{R P}) \geqslant \min (R P),
$$

and the relaxation Theorem 3 shows that

$$
\inf (P)=\min (R P) .
$$

\section{Numerical simulations}

In this section we address the numerical resolution of the problem $(\widetilde{R P})$ in accordance with Conjecture 1 for $a_{\alpha}(t, x)=1$ and $a_{\beta}(t, x)=1$. We first describe the minimization algorithm and then present some numerical experiments.

\subsection{Minimization algorithm}

We briefly present the resolution of the relaxed problem $(\widetilde{R P})$ using a gradient descent method. To this end, we compute the first variation of the cost function.

For any $\eta \in \mathbb{R}^{+}, \eta \ll 1$, and any $s_{1} \in L^{\infty}((0, T) \times \Omega)$, we associate with the perturbation $s^{\eta}=s+\eta s_{1}$ of $s$ the derivative of $\tilde{I}$ with respect to $s$ in direction $s_{1}$ as follows:

$$
\frac{\partial \widetilde{I}(s)}{\partial s} \cdot s_{1}=\lim _{\eta \rightarrow 0} \frac{\tilde{I}\left(s+\eta s_{1}\right)-\widetilde{I}(s)}{\eta} .
$$

We obtain the following result.

THEOREM 4 If $\left(u_{0}, u_{1}\right) \in\left(H^{2}(\Omega) \cap H_{0}^{1}(\Omega)\right) \times H_{0}^{1}(\Omega)$, then the derivative of $\widetilde{I}$ with respect to $s$ in any direction $s_{1}$ exists and takes the form

$$
\frac{\partial \widetilde{I}(s)}{\partial s} \cdot s_{1}=\int_{0}^{T} \int_{\Omega} s_{1}\left(\left(a_{\alpha}-a_{\beta}\right) u_{x}^{2}+(\alpha-\beta) u_{x} p_{x}\right) \mathrm{d} x \mathrm{~d} t
$$

where $u$ is the solution of 21 and $p$ is the solution in $C^{1}\left([0, T] ; H_{0}^{1}(\Omega)\right) \cap C^{1}\left([0, T] ; L^{2}(\Omega)\right)$ of the adjoint problem

$$
\begin{cases}\operatorname{div}\left(p_{t},-[s \alpha+(1-s) \beta] p_{x}\right)=\operatorname{div}\left(u_{t}, a(t, x, s) u_{x}\right) & \text { in }(0, T) \times \Omega \\ p=0 & \text { on }(0, T) \times \partial \Omega, \\ p(T, x)=0, \quad p_{t}(T, x)=u_{t}(T, x) & \text { in } \Omega\end{cases}
$$

Sketch of proof. Let us explain briefly how we obtain the expression 22, We introduce the lagrangian

$$
\mathcal{L}(s, \phi, \psi)=\int_{0}^{T} \int_{\Omega}\left(\phi_{t}^{2}+a(t, x, s) \phi_{x}^{2}\right) \mathrm{d} x \mathrm{~d} t+\int_{0}^{T} \int_{\Omega}\left[\phi_{t t}-\operatorname{div}\left([\alpha s+\beta(1-s)] \phi_{x}\right)\right] \psi \mathrm{d} x \mathrm{~d} t
$$


for any $s \in L^{\infty}((0, T) \times \Omega), \phi \in C\left([0, T] ; H^{2}(\Omega) \cap H_{0}^{1}(\Omega)\right) \cap C^{1}\left([0, T] ; H_{0}^{1}(\Omega)\right)$ and $\psi \in$ $C\left([0, T] ; H_{0}^{1}(\Omega)\right) \cap C^{1}\left([0, T] ; L^{2}(\Omega)\right)$ and then write formally

$$
\frac{d \mathcal{L}}{d s} \cdot s_{1}=\frac{\partial}{\partial s} \mathcal{L}(s, \phi, \psi) \cdot s_{1}+\left\langle\frac{\partial}{\partial \phi} \mathcal{L}(s, \phi, \psi), \frac{\partial \phi}{\partial s} \cdot s_{1}\right\rangle+\left\langle\frac{\partial}{\partial \psi} \mathcal{L}(s, \phi, \psi), \frac{\partial \psi}{\partial s} \cdot s_{1}\right\rangle .
$$

The first term is

$$
\frac{\partial}{\partial s} \mathcal{L}(s, \phi, \psi) \cdot s_{1}=\int_{0}^{T} \int_{\Omega} s_{1}\left(\left(a_{\alpha}-a_{\beta}\right) \phi_{x}^{2}+(\alpha-\beta) \phi_{x} \psi_{x}\right) \mathrm{d} x \mathrm{~d} t
$$

for any $s, \phi, \psi$, whereas the third term is equal to zero if $\phi=u$ the solution of (21). We then determine the solution $p$ so that, for all $\phi \in C\left([0, T] ; H^{2}(\Omega) \cap H_{0}^{1}(\Omega)\right) \cap C^{1}\left([0, T] ; H_{0}^{1}(\Omega)\right)$, we have

$$
\left\langle\frac{\partial}{\partial \phi} \mathcal{L}(s, \phi, p), \frac{\partial \phi}{\partial s} \cdot s_{1}\right\rangle=0
$$

which leads to the formulation of the adjoint problem 23. Next, writing $\widetilde{I}(s)=\mathcal{L}(s, u, p)$, we obtain 22, from 24.

In order to take into account the volume constraint on $s$, we introduce the Lagrange multiplier function $\gamma \in L^{\infty}((0, T), \mathbb{R})$ and the functional

$$
\widetilde{I}_{\gamma}(s)=\widetilde{I}(s)+\int_{0}^{T} \gamma(t) \int_{\Omega} s(t, x) \mathrm{d} x \mathrm{~d} t .
$$

Using Theorem 4 , we find that the derivative of $\widetilde{I}_{\gamma}$ is

$$
\frac{\partial \widetilde{I}_{\gamma}(s)}{\partial s} \cdot s_{1}=\int_{0}^{T} \int_{\Omega} s_{1}\left(\left(a_{\alpha}-a_{\beta}\right) u_{x}^{2}+(\alpha-\beta) u_{x} p_{x}\right) \mathrm{d} x \mathrm{~d} t+\int_{0}^{T} \gamma(t) \int_{\Omega} s_{1} \mathrm{~d} x \mathrm{~d} t,
$$

which permits defining the following descent direction:

$$
s_{1}(x, t)=-\left(\left(a_{\alpha}-a_{\beta}\right) u_{x}^{2}+(\alpha-\beta) u_{x} p_{x}+\gamma(t)\right), \quad \forall x \in \Omega, \forall t \in(0, T) .
$$

Consequently, for any $\eta \in L^{\infty}\left(\Omega \times(0, T) ; \mathbb{R}^{+}\right)$with $\|\eta\|_{L^{\infty}(\Omega \times(0, T))}$ small enough, we have $\widetilde{I}_{\gamma}\left(s+\eta s_{1}\right) \leqslant \widetilde{I}_{\gamma}(s)$. The multiplier function $\gamma$ is then determined in order that $\left\|s+\eta s_{1}\right\|_{L^{1}(\Omega)}=$ $V_{\alpha}|\Omega|$ for any $\eta \in L^{\infty}\left(\Omega \times(0, T) ; \mathbb{R}^{+}\right)$, leading to

$$
\gamma(t)=\frac{\left(\int_{\Omega} s(t, x) \mathrm{d} x-V_{\alpha}|\Omega|\right)-\int_{\Omega} \eta(t, x)\left(\left(a_{\alpha}-a_{\beta}\right) u_{x}^{2}+(\alpha-\beta) u_{x} p_{x}\right) \mathrm{d} x}{\int_{\Omega} \eta(t, x) \mathrm{d} x}
$$

for all $t \in(0, T)$. Finally, the function $\eta$ is chosen so that $s+\eta s_{1} \in[0,1]$ for all $x \in \Omega$ and $t \in(0, T)$. A simple and efficient choice consists in taking $\eta(t, x)=\varepsilon s(t, x)(1-s(t, x))$ for all $x \in \Omega$ and $t \in(0, T)$ with $\varepsilon$ small real positive. Consequently, the descent algorithm to solve numerically the relaxed problem $(\widetilde{R P})$ may be structured as follows :

Let $\Omega \subset \mathbb{R}^{N},\left(u_{0}, u_{1}\right) \in\left(H^{2}(\Omega) \cap H_{0}^{1}(\Omega)\right) \times H_{0}^{1}(\Omega), L \in(0,1), T>0$, and $\varepsilon<1, \varepsilon_{1} \ll 1$ be given. 
- Initialize the density function $s^{0} \in L^{\infty}(\Omega,(0,1))$.

- For $k \geqslant 0$, iterate until convergence (i.e. $\left.\left|\widetilde{I}\left(s^{k+1}\right)-\widetilde{I}\left(s^{k}\right)\right| \leqslant \varepsilon_{1}\left|\widetilde{I}\left(s^{0}\right)\right|\right)$ as follows:

- Compute the solution $u_{s^{k}}$ of (21) and then the solution $p_{s^{k}}$ of [23), both corresponding to $s=s^{k}$.

- Compute the descent direction $s_{1}^{k}$ defined by 25 where the multiplier $\gamma^{k}$ is defined by 26 .

- Update the density function in $\Omega$ :

$$
s^{k+1}=s^{k}+\varepsilon s^{k}\left(1-s^{k}\right) s_{1}^{k}
$$

with $\varepsilon \in \mathbb{R}^{+}$small enough in order to ensure the decrease of the cost function and $s^{k+1} \in$ $L^{\infty}(\Omega \times(0, T) ;[0,1])$.

\subsection{Numerical experiments in the quadratic case}

In this section, we present some numerical simulations for $\Omega=(0,1)$ in the quadratic case, i.e. $\left(a_{\alpha}, a_{\beta}\right)=(1,1)$. We highlight that the numerical resolution of the descent algorithm is a priori difficult because the descent direction (25) depends on the derivative of $u$ and $p$, both solving a wave equation with space and time coefficients only in $L^{\infty}\left((0, T) \times \Omega ; \mathbb{R}_{+}^{\star}\right)$. To the knowledge of the authors, there does not exist any numerical analysis for this kind of equation. We use a $C^{0}$-finite element approximation for $u$ and $p$ with respect to $x$ and a finite difference centered approximation with respect to $t$. Moreover, we add a vanishing viscosity and dispersive term of the type $\epsilon^{2} \operatorname{div}\left([s \alpha+(1-s) \beta] u_{x t t}\right)$ with $\epsilon$ of order of $h$, the space discretization parameter. This term has the effect of regularizing the descent term 25 and leads to a convergent algorithm. Finally, this provides an implicit and unconditionally stable scheme, consistent with (21) and (23), and of order two in time and space.

Below, we treat the following two simple and smooth initial conditions on $\Omega=(0,1)$ :

- CASE 1: $u_{0}(x)=\sin (\pi x), u_{1}(x)=0$;

- CASE 2: $u_{0}(x)=\exp ^{-80(x-0.5)^{2}}, u_{1}(x)=0$,

and we discuss the results depending on the values of $\alpha, \beta$ and $V_{\alpha}$. Results are obtained with $h=$ $d t=10^{-2}, \varepsilon_{1}=10^{-5}, s^{0}(t, x)=V_{\alpha}$ on $[0, T] \times \Omega$ and $\varepsilon=10^{-2}$ (see the algorithm).

7.2.1 Case 1. We first consider Case 1 with $T=2$ and $(\alpha, \beta)=(1,1.1)$. Figure 5 depicts the iso-values of the optimal limit density $s^{\lim }$ (obtained at the convergence of the descent algorithm) for $V_{\alpha}=0.3$ (top) and $V_{\alpha}=0.5$ (bottom) respectively. For these values of $\alpha$ and $\beta$, we observe that the limit densities are characteristic functions taking either the value 0 or 1 . As a consequence, the relaxed problem $(\widetilde{R P})$ coincides with the original one $(P)$, which is well-posed in the class of characteristic functions. This validates Conjecture 1 in this case. Moreover, we observe that the limit densities are independent of the choice of the initialization $s^{0}$. This suggests that $\widetilde{I}$ admits a unique minimum.

Figure 6 represents the corresponding evolution of the energy $E(t)=\frac{1}{2} \int_{\Omega}\left(y_{t}^{2}+[s \alpha+\right.$ $\left.(1-s) \beta] y_{x}^{2}\right) \mathrm{d} x$ with respect to $t$. Due to the time dependence of the coefficients of the state equation, the system is not conservative nor necessarily dissipative.

Results are qualitatively different if we now consider a larger gap $\beta-\alpha$. Figures 7 and 8 represent the result obtained with $(\alpha, \beta)=(1,6)$. We observe that the limit densities are no more characteristic functions and take values in $(0,1)$. This clearly indicates that the original problem 

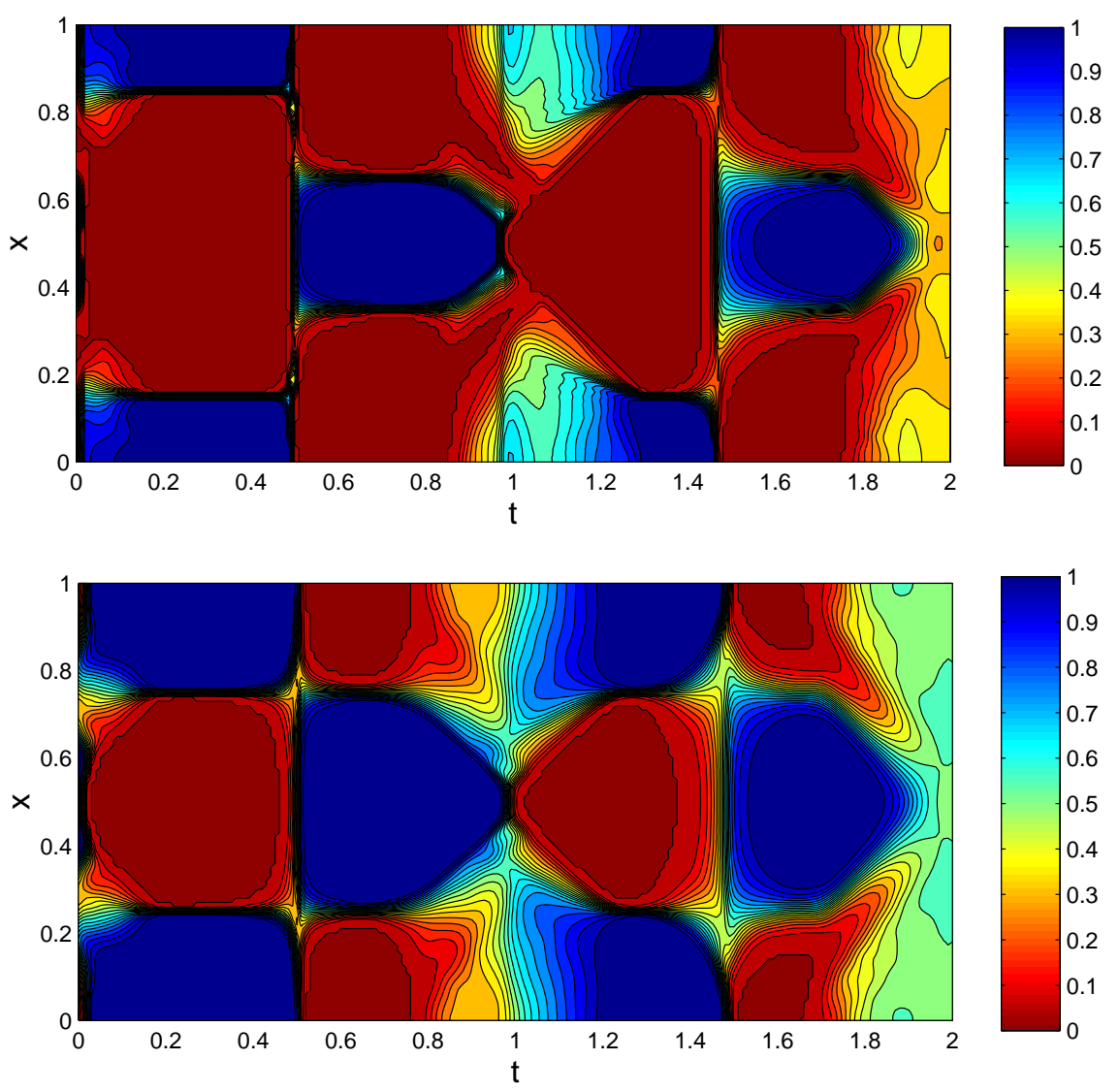

FIG. 5. Case $1, T=2,(\alpha, \beta)=(1,1.1)$. Iso-values of the limit density. Top: $V_{\alpha}=0.3, \tilde{I}\left(s^{\lim }\right) \approx 9.7451$. Bottom: $V_{\alpha}=0.5, \tilde{I}\left(s^{\lim }\right) \approx 9.5613$.
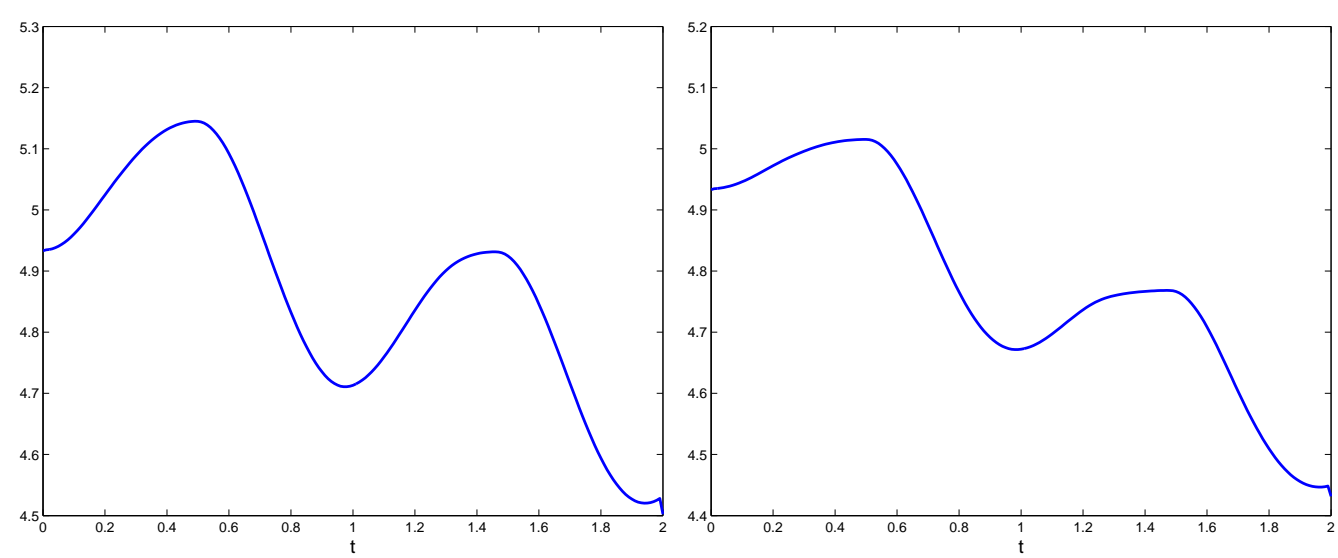

FIG. 6. Case $1, T=2,(\alpha, \beta)=(1,1.1)$. $E(t)$ vs. $t$. Left: $V_{\alpha}=0.3, \widetilde{I}\left(s^{\lim }\right) \approx 9.7451$. Right: $V_{\alpha}=0.5, \widetilde{I}\left(s^{\lim }\right) \approx 9.5613$. 

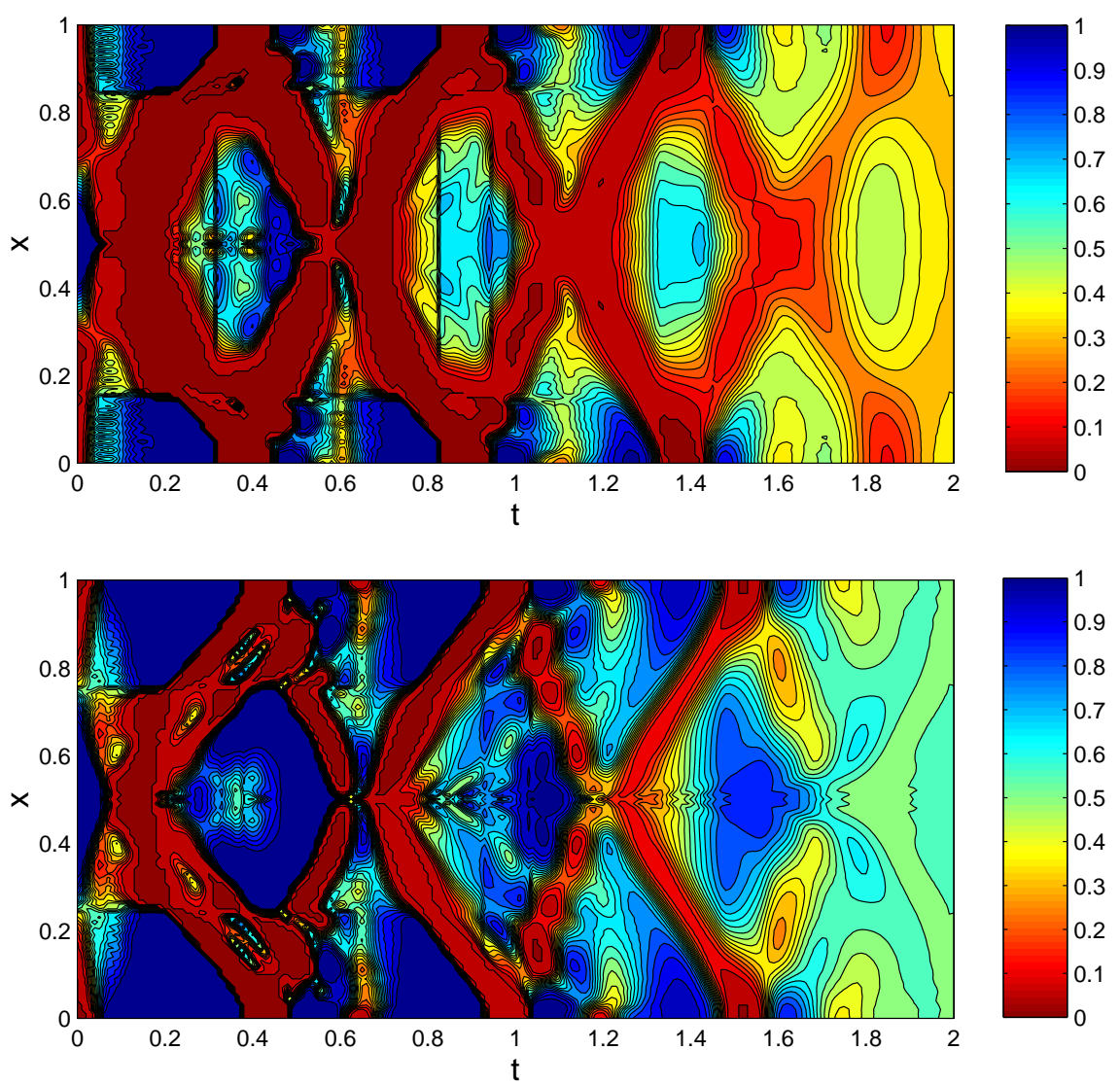

FIG. 7. Case $1, T=2,(\alpha, \beta)=(1,6)$. Iso-values of the limit density. Top: $V_{\alpha}=0.3, \tilde{I}\left(s^{\lim }\right) \approx 7.9567$. Bottom: $V_{\alpha}=0.5$, $\tilde{I}\left(s^{\lim }\right) \approx 6.1439$.
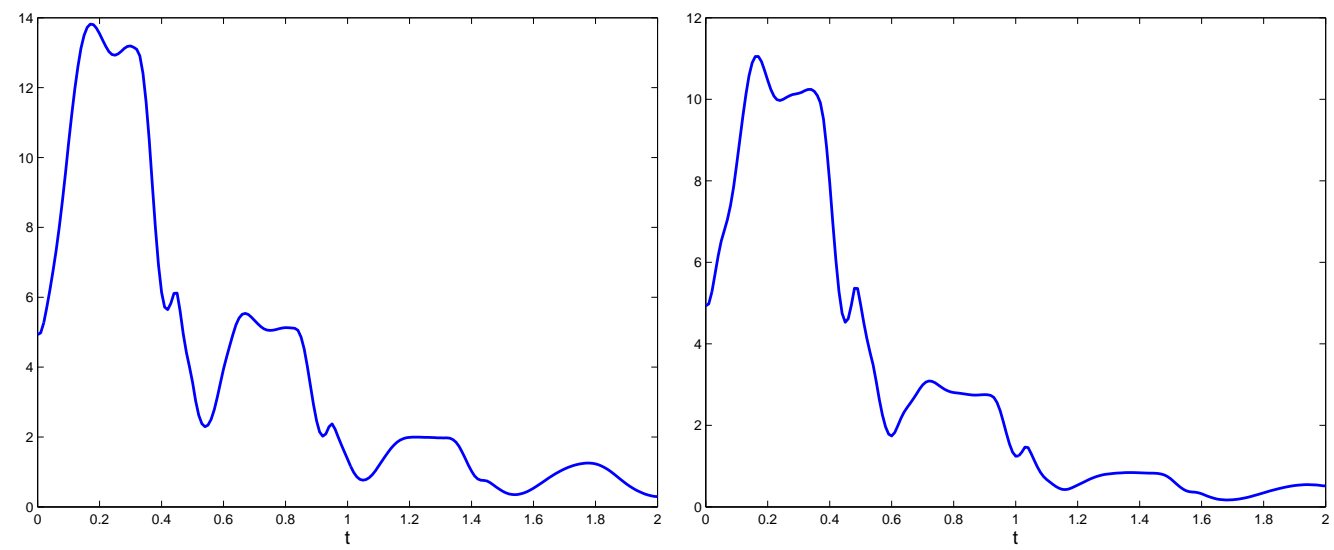

FIG. 8. Case $1, T=2,(\alpha, \beta)=(1,2) . E(t)$ vs. $t$. Left: $V_{\alpha}=0.3, \widetilde{I}\left(s^{\lim }\right) \approx 7.9567$. Right: $V_{\alpha}=0.5, \widetilde{I}\left(s^{\lim }\right) \approx 6.1439$. 

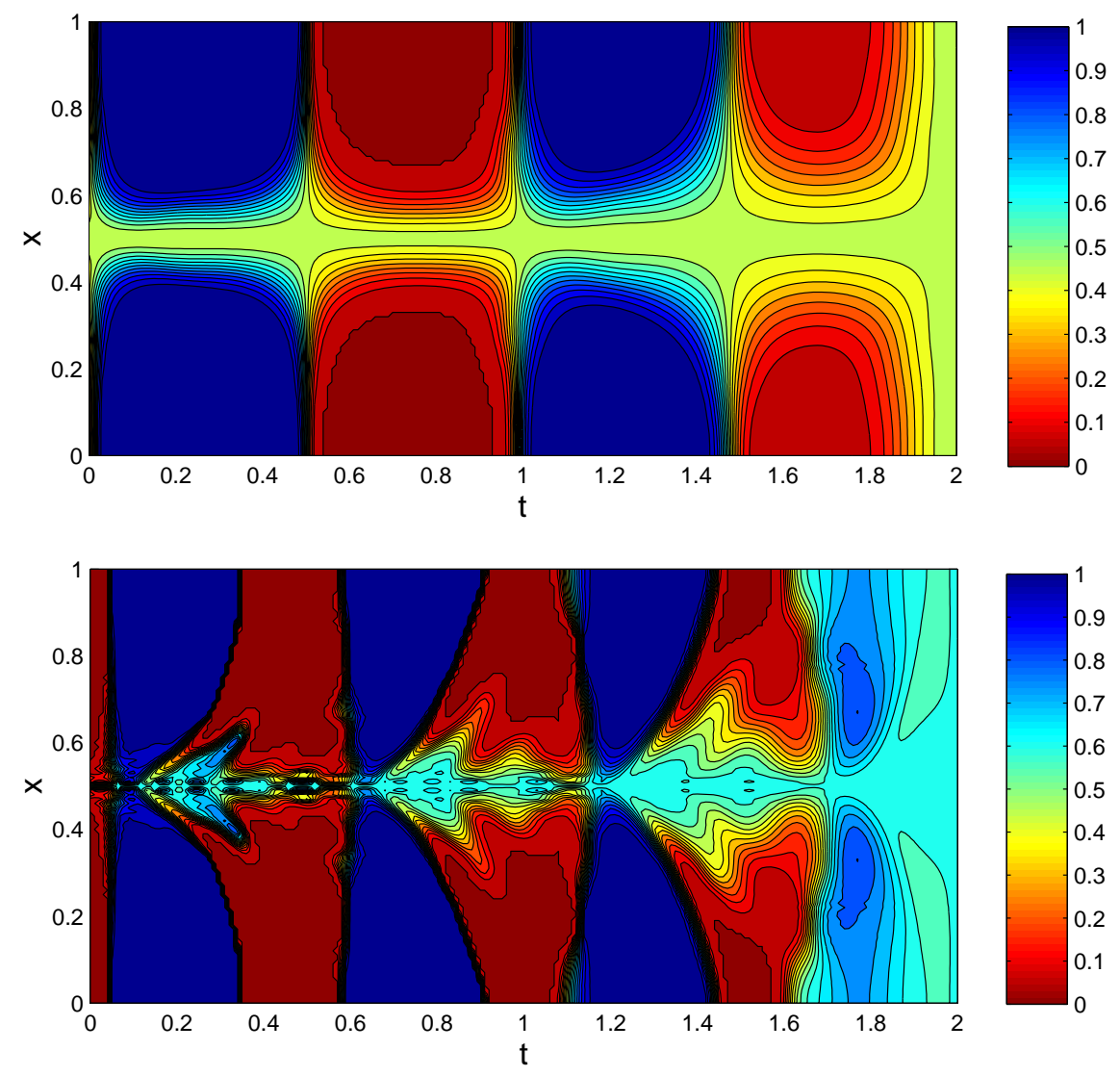

FIG. 9. Case 1 with the volume constraint $277, T=2, V_{\alpha}=0.5$. Iso-value of the limit density. Top: $(\alpha, \beta)=(1,1.1)$, $\widetilde{I}\left(s^{\lim }\right) \approx 9.2147$. Bottom: $(\alpha, \beta)=(1,6), \tilde{I}\left(s^{\lim }\right) \approx 4.3109$.

may not be well-posed and justifies the search of a relaxed formulation. We also observe that this property depends on the value of $V_{\alpha}$ : for $V_{\alpha}$ or $1-V_{\alpha}$ arbitrarily small, numerical simulation leads to bi-valued limit densities for all $\alpha$ and $\beta$.

We have also observed that as soon as the gap is large enough, the limit of the density depends on the initialization $s^{0}$, highlighting the existence of several infima for $\widetilde{I}$. We found that the choice of $s^{0}$ constant on $(0, T) \times \Omega$-which has the advantage of not favoring any distribution between $\alpha$ and $\beta$-leads to the lowest value of $\widetilde{I}\left(s^{\lim }\right)$. Moreover, for this choice, the algorithm appears robust, stable and convergent with respect to the discretization parameters $h$ and $\Delta t$. Under these circumstances, we suspect that the infimum of $(\widetilde{R P})$ (see Lemma 2 ) is in fact a minimum.

We remark that the relaxation analysis and the results presented in the previous sections are unchanged if we consider the weaker volume constraint:

$$
\int_{0}^{T} \int_{\Omega} s(t, x) \mathrm{d} x \mathrm{~d} t \leqslant V_{\alpha}|\Omega| T .
$$


Figure 9 depicts the limit densities for $V_{\alpha}=0.5$ for $(\alpha, \beta)=(1,1.1)$ (top) and $(\alpha, \beta)=(1,6)$ (bottom) respectively. Furthermore, as expected, these densities lead to a better distribution of materials: we obtain $\widetilde{I}\left(s^{\lim }\right) \approx 9.2147$ and $\widetilde{I}\left(s^{\lim }\right) \approx 3.4709$ respectively $\left(\mathrm{cf} . \widetilde{I}\left(s^{\lim }\right) \approx 9.5613\right.$ and $\widetilde{I}\left(s^{\lim }\right) \approx 6.1439$ for the initial volume constraint $\int_{\Omega} s(t, x) \mathrm{d} x \leqslant V_{\alpha}|\Omega|$ for all $\left.t\right)$.

7.2.2 Case 2. We now present some results for the second case. Similarly to the first case, the optimal density takes values in $(0,1)$ if and only if the gap $\beta-\alpha$ is large enough. The pictures also clearly highlight that the optimal distribution is related to the propagation of the components of the solution on the cylinder $(0, T) \times(0,1)$. For this case, we observe that the two volume constraints give similar results on the density and the optimal cost (see Figures 10 and 12). Furthermore, in the case $(\alpha, \beta)=(1,10)$, we observe in Figure 13 the strong damping mechanism of the optimal distribution and explain why, for $t$ sufficiently large, the value of the cost function is less sensitive to the density $s$ (i.e. for $t$ large, the variations of $s$ with respect to $x$ and $t$ are low).

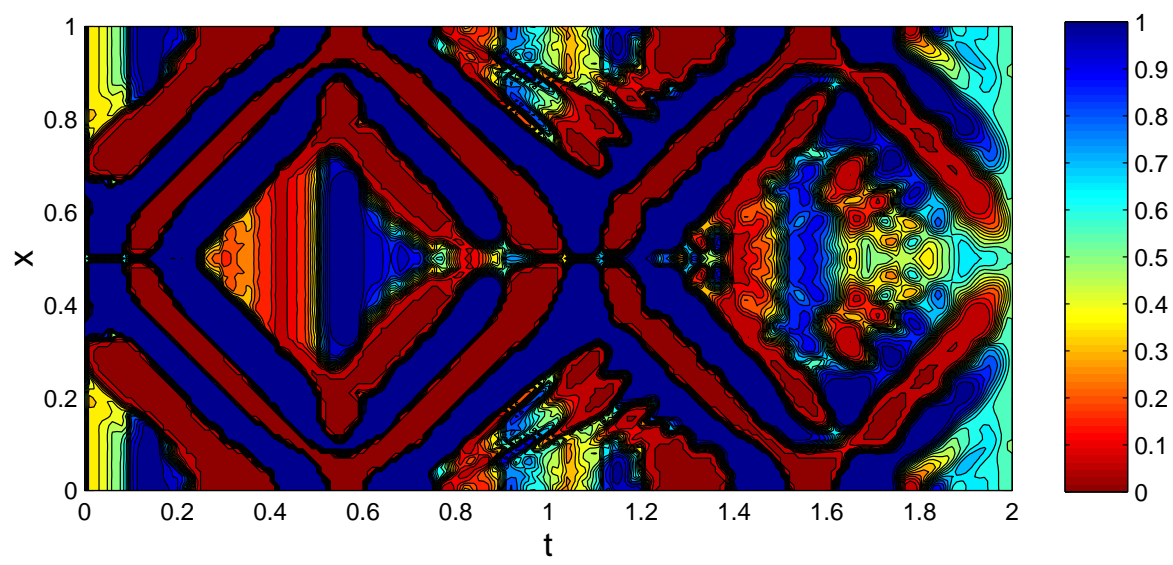

FIG. 10. Case $2, T=2,(\alpha, \beta)=(1,1.1)$. Iso-value of the limit density. $V_{\alpha}=0.5, \widetilde{I}\left(s^{\lim }\right) \approx 15.48$.

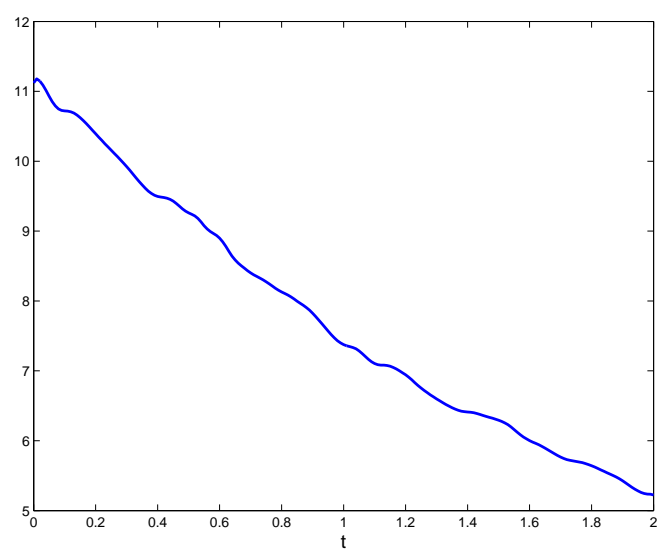

FIG. 11. Case $2, T=2,(\alpha, \beta)=(1,1.1) . E(t)$ vs. $t . V_{\alpha}=0.5, \widetilde{I}\left(s^{\lim }\right) \approx 15.48$ 


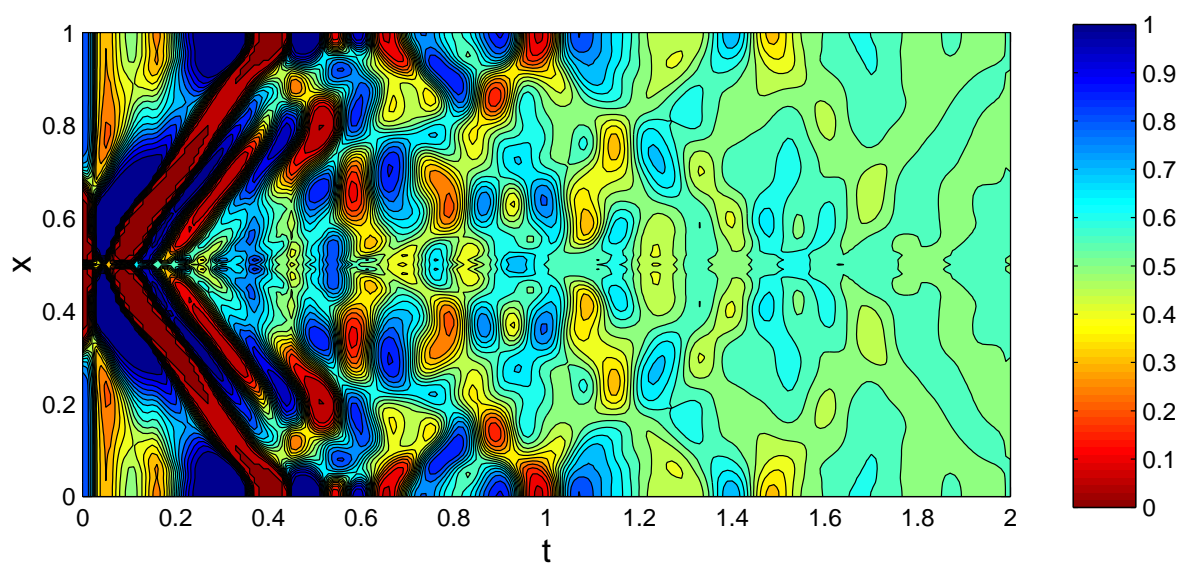

FIG. 12. Case $2, T=2,(\alpha, \beta)=(1,6)$. Iso-value of the limit density. $V_{\alpha}=0.5, \widetilde{I}\left(s^{\lim }\right) \approx 4.5414$.

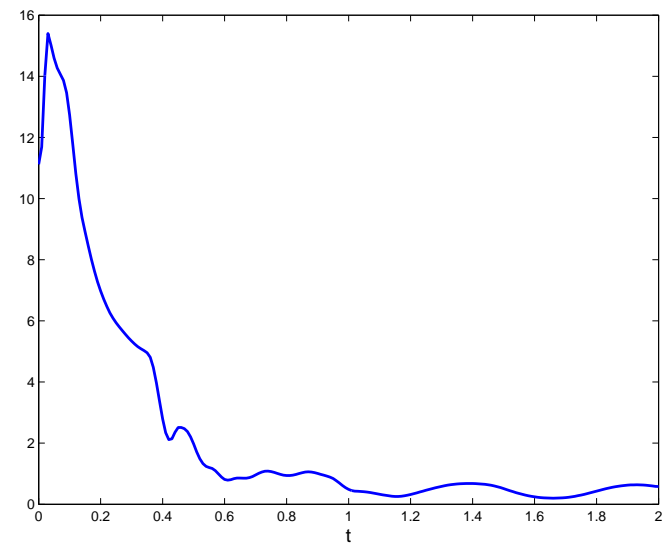

FIG. 13. Case $2, T=2,(\alpha, \beta)=(1,6) . E(t)$ vs. $t . V_{\alpha}=0.5, \widetilde{I}\left(s^{\lim }\right) \approx 4.5414$.

7.2.3 Construction of a characteristic density associated to $s$ lim. In the case where the optimal density $s^{\lim }$ is not in $L^{\infty}((0, T) \times \Omega ;\{0,1\})$, one may associate with $s^{\lim }$ a characteristic function $s^{\text {pen }} \in L^{\infty}((0, T) \times \Omega ;\{0,1\})$ whose cost $\widetilde{I}\left(s^{\text {pen }}\right)$ is arbitrarily near to $\widetilde{I}\left(s^{\lim }\right)$. Following [21], one may proceed as follows: we first decompose the cylinder $(0, T) \times \Omega$ into $M \times N$ cells such that $(0, T) \times \Omega=\bigcup_{i=1}^{M}\left[t_{i}, t_{i+1}\right] \times \bigcup_{j=1}^{N}\left[x_{j}, x_{j+1}\right]$. Then we associate with each cell the mean value $m_{i, j} \in[0,1]$ defined by

$$
m_{i, j}=\frac{1}{\left(t_{i+1}-t_{i}\right)\left(x_{j+1}-x_{j}\right)} \int_{t_{i}}^{t_{i+1}} \int_{x_{j}}^{x_{j+1}} s^{\lim }(t, x) \mathrm{d} x \mathrm{~d} t .
$$

Finally, we define the function $s_{M, N}^{\text {pen }}$ in $L^{\infty}([0, T] \times \Omega)$ by

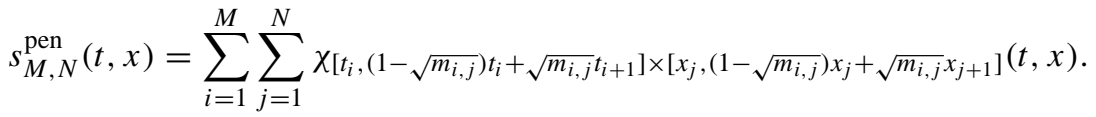



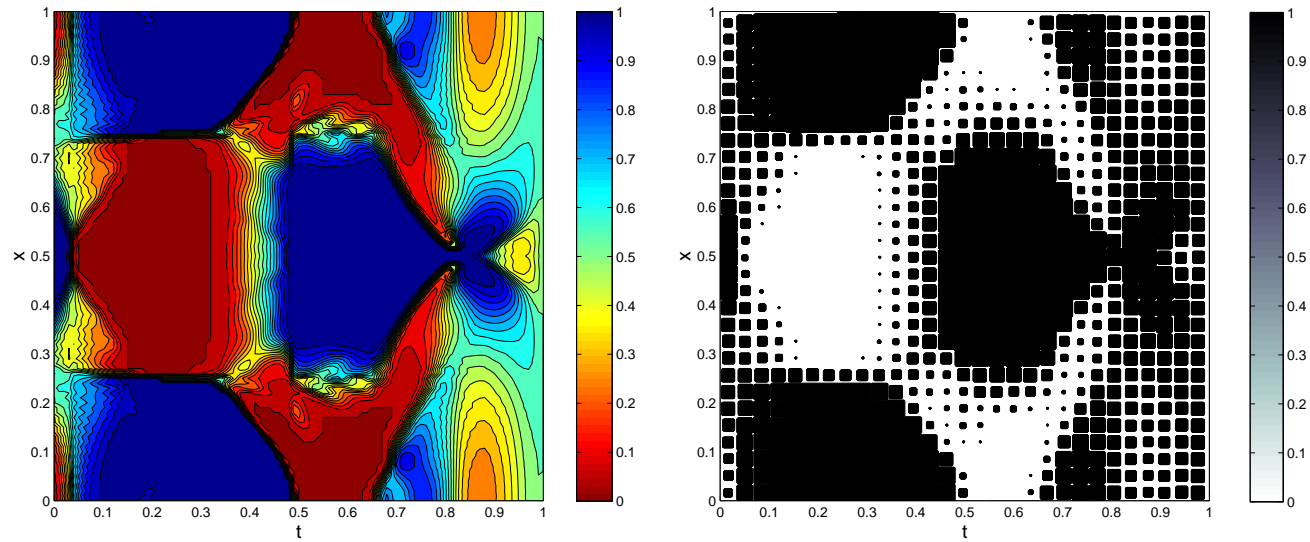

FIG. 14. Case $1, T=1,(\alpha, \beta)=(1,2) . V_{\alpha}=0.5, s_{30,30}^{\text {pen }}, \widetilde{I}\left(s^{\lim }\right) \approx 4.7584, \widetilde{I}\left(s_{30,30}^{\text {pen }}\right) \approx 5.62$.

We easily check that $\left\|s_{M, N}^{\text {pen }}\right\|_{L^{1}((0, T) \times \Omega)}=\| s^{\lim \|_{L^{1}((0, T) \times \Omega)}}$ for all $M, N>0$. Thus, the bi-valued function $s_{M, N}^{\mathrm{pen}}$ takes advantage of the information codified in the density $s^{\lim }$.

In order to illustrate this, we consider Case 1 with $T=1,(\alpha, \beta)=(1,2)$ and $V_{\alpha}=0.5$. Figure 14 depicts the corresponding optimal density $s^{\lim }$ and the associated function $s_{M, N}^{\text {pen }}$ for $M=$ $N=30$. We obtain $\widetilde{I}\left(s_{30,30}^{\text {pen }}\right)=5.62$ and $\widetilde{I}\left(s^{\lim }\right)=4.7584$ respectively. By letting $M$ and $N$ go to infinity, we expect convergence to the value $\widetilde{I}\left(s^{\lim }\right)$ and then construct a minimizing sequence of domains $\omega_{M, N}$ such that $\chi_{\omega_{\infty, \infty}}$ is the infimum for $I$ (see Table 1]. We refer to [19] for more examples.

TABLE 1

Case $1, T=1,(\alpha, \beta)=(1,3), V_{\alpha}=0.5$. Values of the cost function $\widetilde{I}\left(s_{M, N}^{\mathrm{pen}}\right)$

\begin{tabular}{lccccc}
\hline$M=N$ & 10 & 20 & 30 & 40 & 50 \\
\hline$\widetilde{I}\left(s_{M, N}^{\mathrm{pen}}\right)$ & 7.45 & 6.21 & 5.62 & 5.09 & 4.93 \\
\hline
\end{tabular}

\section{Acknowledgements}

The first and third authors are partially supported by project MTM2004-07114 from Ministerio de Educación y Ciencia (Spain), by project PAI05-029 from JCCM (Castilla - La Mancha) and the first author by $\mathrm{PhD}$ grant 03/034 of JCCM. We would like to thank various referees for helpful comments and constructive criticism which led to an improved version of the manuscript.

\section{REFERENCES}

1. Allaire, G. Shape Optimization by the Homogenization Method. Springer (2002). Zbl 0990.35001 MR 1859696 
2. Aranda, E., \& Pedregal, P. Constrained envelope for a general class of design problems. Disc. Cont. Dyn. Syst., Suppl. Vol., (2003), 30-41. Zbl 1062.49011 MR 2018097

3. Bellido, J. C., \& Donoso, A. An optimal design problem in wave propagation. J. Optim. Theory Appl. 134 (2007), 339-352. MR 2332468

4. Bendsøe, MP., \& Sigmund, O. Topology Optimization: Theory, Methods and Aplications. Springer, Berlin (2003). Zbl 1059.74001 MR 2008524

5. CAstro, C., \& CoX, S. Achieving arbitrarily large decay in the damped wave equation. SIAM J. Control Optim. 39 (2001), 1748-1755. Zbl 0983.35095 MR 1825863

6. Chambolle, A., \& SAntosa, F. Control of the wave equation by time-dependent coefficient. ESAIMCOCV 8 (2002) 375-392. Zbl 1073.35032 MR 1932956

7. Cherkaev, A. Variational Methods for Structural Optimization. Springer, New York (2000). Zbl 0956.74001 MR 1763123

8. Dacorogna, B. Direct Method in the Calculus of Variations. Springer (1989). Zbl 0703.49001 MR 0990890

9. Donoso, A., \& Pedregal, P. Optimal design of 2-D conducting graded materials by minimizing quadratic functionals in the field. Struct. Multidiscip. Optim. 30 (2005), 360-367. MR 2176301

10. Freitas, P. Optimizing the rate of decay of solutions of the wave equation using genetic algorithms: a counterexample to the constant damping conjecture. SIAM J. Control Optim. 37 (1999), 376-387. Zbl 0999.35010 MR 1655858

11. GRABOVSKY, Y. Optimal design problems for two-phase conducting composites with weakly discontinuous objective functionals. Adv. Appl. Math. 27 (2001), 683-704. Zbl 1001.49002 MR 1867929

12. Hébrard, P., \& Henrot, A. Optimal shape and position of the actuators for the stabilization of a string. System Control Lett. 48 (2003), 199-209. Zbl pre05055493 MR 2020637

13. Lions, J.-L., \& Magenes, E. Non-Homogeneous Boundary Value Problems and Applications. Vol. I, Springer (1972). Zbl 0223.35039 MR 0350177

14. Lipton, R., \& Velo, A. P. Optimal design of gradient fields with applications to electrostatics. Nonlinear Partial Differential Equations and Their Applications, Collège de France Seminar, Vol. XIV, D. Cioranescu and J.-L. Lions (eds.), North-Holland (2002), 509-532. Zbl 1080.78003 MR 1936008

15. LURIE, K. A. Control in the coefficients of linear hyperbolic equations via spatio-temporal components. Homogenization, V. Berdichevsky et al. (eds.), World Sci., Singapore (1999), 285-315. Z Zbl 1035.78021 MR 1792692

16. LURIE, K. A. The problem of the effective parameters of a mixture of two isotropic dielectrics distributed in space-time and the conservation law of wave impedance in one-dimensional wave propagation. Proc. R. Soc. London A 454 (1998), 1767-1779. Zbl 0915.35102 MR 1640028

17. LURIE, K. A. A stable spatio-temporal $G$-closure and $G_{m}$-closure of a set of isotropic dielectrics with respect to one-dimensional wave propagation. Wave Motion 40 (2004), 95-110. Zbl pre02202502 MR 2069904

18. MAESTRE, F. In progress.

19. Maestre, F., MÜNCh, A., \& Pedregal, P. A spatio-temporal design problem for a damped wave equation. SIAM J. Appl. Math. 68 (2007), 109-132. MR 2357775

20. Milton, G. W. The Theory of Composites. Cambridge Univ. Press (2002). Zbl 0993.74002 MR 1899805

21. Münch, A., Pedregal, P., \& Periago, F. Optimal design of the damping set for the stabilization of the wave equation. J. Differential Equations 231 (2006), 331-358. Zbl 1105.49005 MR 2287890

22. Murat, F. Contre-exemples pour divers problèmes où le contrôle intervient dans les coefficients. Ann. Mat. Pura Appl. 112 (1977), 49-68. Zbl 0349.49005 MR 0438205

23. Pedregal, P. Vector variational problems and applications to optimal design. ESAIM-COCV 15 (2005), 357-381. Zbl 1089.49022 MR 2148849 
24. Pedregal, P. Constrained quasiconvexification of the square of the gradient of the state in optimal design. Quart. Appl. Math. 62 (2004), 459-470. Zbl 1086.49013 MR 2086039

25. Pedregal, P. Optimal design in 2-D conductivity for quadratic functionals in the field. Nonlinear Homogenization and its Applications to Composites, Polycrystals and Smart Materials (Warszawa, 2003), Kluwer (2004), 229-246. MR 2268907

26. Pedregal, P. Optimal design in two-dimensional conductivity for a general cost depending on the field. Arch. Ration. Mech. Anal. 182 (2006), 367-385. Zbl 1104.74052 MR 2276496

27. TARTAR, L. Remarks on optimal design problems. Calculus of Variations, Homogenization and Continuum Mechanics, G. Buttazzo et al. (eds.), World Sci., Singapore (1994), 279-296. Zbl 0884.49015 MR 1428706 